\title{
Decreased somatic hypermutation induces an impaired peripheral B cell tolerance checkpoint
}

\author{
Tineke Cantaert, ${ }^{1}$ Jean-Nicolas Schickel, ${ }^{1}$ Jason M. Bannock, ${ }^{1}$ Yen-Shing Ng, ${ }^{1}$ Christopher Massad, ${ }^{1}$ Fabien R. Delmotte, ${ }^{1}$ \\ Natsuko Yamakawa, ${ }^{1}$ Salome Glauzy, ${ }^{1}$ Nicolas Chamberlain, ${ }^{1}$ Tuure Kinnunen, ${ }^{1}$ Laurence Menard, ${ }^{1}$ Aubert Lavoie, ${ }^{2}$ \\ Jolan E. Walter, ${ }^{3,4,5}$ Luigi D. Notarangelo, ${ }^{6}$ Julie Bruneau, ${ }^{7}$ Waleed Al-Herz, ${ }^{8}$ Sara Sebnem Kilic, ${ }^{9}$ Hans D. Ochs, ${ }^{10}$ \\ Charlotte Cunningham-Rundles, ${ }^{11}$ Mirjam van der Burg, ${ }^{12}$ Taco W. Kuijpers, ${ }^{13}$ Sven Kracker, ${ }^{14}$ Hideo Kaneko, ${ }^{15}$ \\ Yujin Sekinaka, ${ }^{16}$ Shigeaki Nonoyama, ${ }^{16}$ Anne Durandy, ${ }^{14}$ and Eric Meffre ${ }^{1}$
}

'Department of Immunobiology, Yale University School of Medicine, New Haven, Connecticut, USA. 'Department of Medicine, Immunology and Allergy Service, Centre Hospitalier de I'Université Laval, Quebec City, Quebec, Canada. ${ }^{3}$ Division of Pediatric Allergy/Immunology, University of South Florida and Johns Hopkins All Children's Hospital, St. Petersburg, Florida, USA. ^Division of Pediatric Allergy/ Immunology, Massachusetts General Hospital for Children, Boston, Massachusetts, USA. ${ }^{5}$ Division of Immunology, Boston Children's Hospital, Harvard Medical School, Boston, Massachusetts, USA. ${ }^{6}$ Division of Immunology and Manton Center for Orphan Disease Research, Children's Hospital Boston, Harvard Medical School, Boston, Massachusetts, USA. ${ }^{7}$ Pathology Department, Hôpital Necker Enfants Malades, Paris, France. ${ }^{8}$ Department of Pediatrics, Al-Sabah Hospital, and Department of Pediatrics, Faculty of Medicine, Kuwait University, Kuwait City, Kuwait. ${ }^{9}$ Uludag University Medical Faculty, Department of Pediatrics, Gorukle - Bursa, Turkey. ${ }^{10}$ Seattle Children's Research Institute and Department of Pediatrics, University of Washington, Seattle, Washington, USA. "Department of Medicine, Mount Sinai Medical Center, New York, New York, USA. ${ }^{2}$ Department of Immunology, Erasmus MC, University Medical Center Rotterdam, Rotterdam, Netherlands. ${ }^{13}$ Department of Pediatric Hematology, Immunology, and Infectious Diseases, Emma Children's Hospital, Academic Medical Center, Amsterdam, Netherlands. ${ }^{14}$ Laboratory of Human Lymphohematopoiesis, INSERM UMR U1163 and Paris Descartes - Sorbonne Paris Cité University, Imagine Institute, Paris, France. ${ }^{15}$ Department of Clinical Research, National Hospital Organization Nagara Medical Center, Cifu, Japan. ${ }^{16}$ Department of Pediatrics, National Defense Medical College, Tokorozawa, Saitama, Japan.

Patients with mutations in AICDA, which encodes activation-induced cytidine deaminase (AID), display an impaired peripheral B cell tolerance. AID mediates class-switch recombination (CSR) and somatic hypermutation (SHM) in B cells, but the mechanism by which AID prevents the accumulation of autoreactive $B$ cells in blood is unclear. Here, we analyzed $B$ cell tolerance in AID-deficient patients, patients with autosomal dominant AID mutations (AD-AID), asymptomatic AICDA heterozygotes ( $\left(\mathrm{ID}^{+/-}\right)$, and patients with uracil $\mathrm{N}$-glycosylase (UNC) deficiency, which impairs CSR but not SHM. The low frequency of autoreactive mature naive B cells in UNG-deficient patients resembled that of healthy subjects, revealing that impaired CSR does not interfere with the peripheral B cell tolerance checkpoint. In contrast, we observed decreased frequencies of SHM in memory B cells from AD-AID patients and AID+/- subjects, who were unable to prevent the accumulation of autoreactive mature naive B cells. In addition, the individuals with AICDA mutations, but not UNGdeficient patients, displayed Tregs with defective suppressive capacity that correlated with increases in circulating $T$ follicular helper cells and enhanced cytokine production. We conclude that SHM, but not CSR, regulates peripheral B cell tolerance through the production of mutated antibodies that clear antigens and prevent sustained interleukin secretions that interfere with Treg function.

\section{Introduction}

Developing B cells with autoreactive B cell receptors (BCRs) generated by random $\mathrm{V}(\mathrm{D}) \mathrm{J}$ recombination are removed at 2 discrete early B cell tolerance checkpoints; a central checkpoint in the bone marrow removes most clones expressing polyreactive and antinuclear autoantibodies, whereas a peripheral B cell tolerance checkpoint prevents the accumulation of autoreactive mature naive B cells (1). This second selection step appears to be regulated by B cell-extrinsic factors such as Tregs, since FOXP3-deficient patients who lack functional Tregs display a defective peripheral B cell tolerance checkpoint (2). In agreement with this observation, decreased Treg numbers in patients with class-switch recombination deficiency (CSR-D) caused by mutations in CD4OL/TNFRSF5

Conflict of interest: The authors have declared that no conflict of interest exists Submitted: September 11, 2015; Accepted: August 30, 2016.

Reference information: / Clin Invest. 2016;126(11):4289-4302. doi:10.1172/JCI84645. or in the AICDA gene encoding activation-induced cytidine deaminase (AID), which mediates CSR and somatic hypermutation (SHM), also correlate with an impaired peripheral B cell tolerance checkpoint (3-9). However, the mechanisms by which AID may affect Treg homeostasis or function remain unknown.

To assess the individual contribution of CSR and SHM to the establishment of peripheral B cell tolerance, we analyzed the frequency of autoreactive mature naive B cells and Treg function in rare uracil $\mathrm{N}$-glycosylase-deficient (UNG-deficient) patients, CSR-D patients with autosomal dominant (AD) AICDA mutations, and healthy asymptomatic individuals carrying a single autosomal recessive AICDA mutation ( $\mathrm{AID}^{+/-}$heterozygotes). Patients lacking UNG, an enzyme that excises from DNA uracils resulting from enzymatic deamination of cytosines by AID, have impaired CSR but functional SHM processes, although with a skewed pattern (3). Patients with the V186X or R190X heterozygous AD mutation in AICDA, which preserves AID enzymatic activity, also have 
impaired CSR (10). The V186X or R190X stop mutations result in the truncation of the AID C-terminal nuclear export signal and cause an accumulation in the nucleus of mutated AID proteins that are unable to catalyze CSR, whereas unmutated AID produced by the normal allele can be shuttled out of the nucleus (11).

We report here that autoreactive B cells are normally counterselected in UNG-deficient patients, demonstrating that CSR and the generation of isotype-switched $\mathrm{B}$ cells are not involved in the regulation of the peripheral B cell tolerance checkpoint. In contrast, altered SHM in AID-deficient patients, AD-AID individuals, and $\mathrm{AID}^{+/-}$heterozygotes resulted in increased numbers of autoreactive mature naive $B$ cells in the periphery and correlated with impaired Treg function that was likely due to elevated cytokine production.

\section{Results}

Abnormal peripheral B cell tolerance checkpoint in subjects carrying heterozygous AICDA mutation(s). We have previously reported that AID-deficient patients display an impaired peripheral B cell tolerance checkpoint (8). However, it is unclear whether this altered selection step results from defective CSR or SHM, since both are catalyzed by AID. In an attempt to discern the single contribution of CSR or SHM to preventing the accumulation of autoreactive B cells in the periphery, we analyzed the Ig repertoire and the reactivity of recombinant antibodies cloned from mature naive B cells from CSR-D patients including 3 UNG-deficient and 4 AD-AID patients with impaired CSR. We also studied 5 asymptomatic $\mathrm{AID}^{+/-}$subjects with a single recessive AICDA mutation and 2 additional AID-deficient patients (8). Repertoire analysis in mature naive $B$ cells from UNG-deficient patients revealed normal frequencies of the $V_{H / 4-34}$ gene (Figure 1A and Supplemental Tables 3-16; supplemental material available online with this article; doi:10.1172/ JCI84645DS1), which is known to encode intrinsically self-reactive cold agglutinin antibodies $(12,13)$. In contrast, we found that $V_{H / 4-34}$ gene segment usage was increased in mature naive $\mathrm{B}$ cells from AID-deficient patients, AD-AID patients, and $\mathrm{AID}^{+/}$heterozygotes, suggesting an abnormal peripheral B cell tolerance checkpoint in subjects carrying AICDA mutation(s) (Figure 1A). We performed ELISA on HEp-2 cell lysates to test the reactivity of recombinant antibodies cloned from mature naive $\mathrm{B}$ cells to determine the functionality of the peripheral B cell tolerance checkpoint $(1,14)$. The analysis of 2 additional AID-deficient patients confirmed our previous observation of increased frequencies of HEp-2-reactive clones, which represented $52.1 \% \pm 7.1 \%$ of the mature naive B cells compared with $20.4 \% \pm 3.6 \%$ in healthy donor (HD) counterparts $(P<0.0001$; Figure 1, B and C, and Supplemental Figure 1) (8). In agreement with abnormal $V_{H / 4-34}$ gene segment usage, the frequency of HEp-2-reactive clones was also increased in $\mathrm{AID}^{+/}$heterozygotes $(36.8 \% \pm 6.0 \%)$ and in $\mathrm{AD}$-AID patients $(42.7 \% \pm 10.0 \%)$, revealing an impaired peripheral B cell tolerance checkpoint (Figure 1, B and C, and Supplemental Figure 1). Peripheral B cell tolerance checkpoint defects were further evidenced in all subjects carrying AICDA mutation(s) by the elevated frequencies of polyreactive clones compared with frequencies in HDs (Figure 1D and Supplemental Figure 2). In addition, the frequencies of antinuclear B cells were also elevated in AID-deficient patients $(13.1 \% \pm 5.4 \%$ in AID-deficient patients compared with $3.3 \% \pm 2.2 \%$ in HDs, $P<0.001$ ) (Figure 1E). Various patterns of HEp-2-reactive antibod- ies that recognized nuclear or cytoplasmic structures are shown in Figure 1F. Of note, the increased self-reactivity in $\mathrm{AID}^{+/-} \mathrm{B}$ cells was less severe than in $\mathrm{AID}^{-/-} \mathrm{B}$ cells, suggesting a gene dosage effect of AICDA on this peripheral $\mathrm{B}$ cell selection step (Figure 1, B and C, and Supplemental Figure 1). In contrast, UNG-deficient patients displayed normal frequencies of HEp-2-reactive (23.8\% $\pm 2.0 \%)$, polyreactive $(10.9 \% \pm 5.3 \%)$, and antinuclear $(1.7 \% \pm 3.0 \%)$ mature naive B cells, demonstrating that impaired CSR and the absence of isotype-switched memory B cells do not affect the establishment of peripheral B cell tolerance (Figure 1, B-E). We conclude that AICDA mutations induce defects in the peripheral $\mathrm{B}$ cell tolerance checkpoint independently of CSR impediments.

Elevated systemic cytokine concentrations correlate with increased homeostatic B cell expansion of autoreactive mature naive B cells. We have previously observed that defects in peripheral B cell tolerance may be associated with increased cytokine production in patients with common variable immunodeficiency (CVID) and in those with SLAM-associated protein (SAP) deficiency $(15,16)$. Serum concentrations of IL-2, IL-6, IL-10, IL-17, IFN- $\gamma$, and IL-21 were increased in AID-deficient patients, while some $\mathrm{AID}^{+/-}$subjects and AD-AID patients displayed increased serum IL-6 and IL-10 or IL-12 and IL-21 concentrations, respectively, although differences did not reach statistical significance for the latter as determined by ANOVA with Dunnett's correction for multiple comparisons (Figure 2A). Although we could only test 2 UNG-deficient patients, they did not show any difference in proinflammatory cytokines compared with HDs, thus correlating with the normal peripheral B cell tolerance checkpoint observed in these UNG-deficient patients (Figure 2A).

$\mathrm{B}$ cell-activating factor (BAFF) is another molecule that controls the number of peripheral $B$ cells and has been associated with peripheral B cell tolerance dysregulation (17). However, the impact of BAFF on the accumulation of autoreactive mature naive B cells remains unclear. While increased serum BAFF concentrations in CD4OL- and AID-deficient patients as well as in CVID patients correlated with an altered peripheral B cell tolerance checkpoint, FOXP3-, SAP-, and DOCK8-deficient patients showed normal serum BAFF concentrations, despite increased frequencies of autoreactive mature naive $\mathrm{B}$ cells $(2,7,8,15,16,18)$. We found that all CSR-D patients, who have no switched memory B cells, had a 2-fold increase in serum BAFF concentrations (Figure 2B). Since the peripheral $\mathrm{B}$ cell tolerance checkpoint is functional in UNGdeficient patients, we can conclude that a 2-fold increase in the serum BAFF concentration does not alter this peripheral B cell selection step. In addition, $\mathrm{AID}^{+/-}$subjects who display a defective peripheral B cell tolerance checkpoint showed normal serum BAFF concentrations, further revealing a lack of correlation between serum BAFF concentrations and the accumulation of autoreactive mature naive $\mathrm{B}$ cells (Figure 2B). Increased serum BAFF concentrations may be associated with increased homeostatic B cell expansion, as previously observed in other patients with primary immunodeficiency (16). Using a quantitative PCR-based assay, we measured the $\kappa$-deletion recombination excision circles (KRECs) to obtain an estimate of the number of divisions undergone by a B cell population, and found an increase in the number of divisions in mature naive $B$ cells from AID-deficient $(2.7 \pm 0.9, P<0.01)$ and AD-AID $(2.9 \pm 0.8, P<0.05)$ patients but not in those from UNG-deficient patients $(2.2 \pm 0.4)$ or $\mathrm{AID}^{+/-}$subjects $(1.6 \pm 0.4)$, thereby revealing 


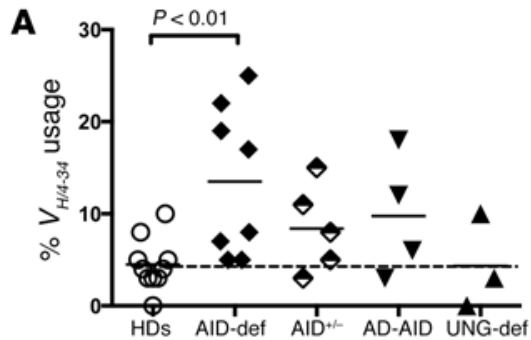

B

Mature naive $B$ cells
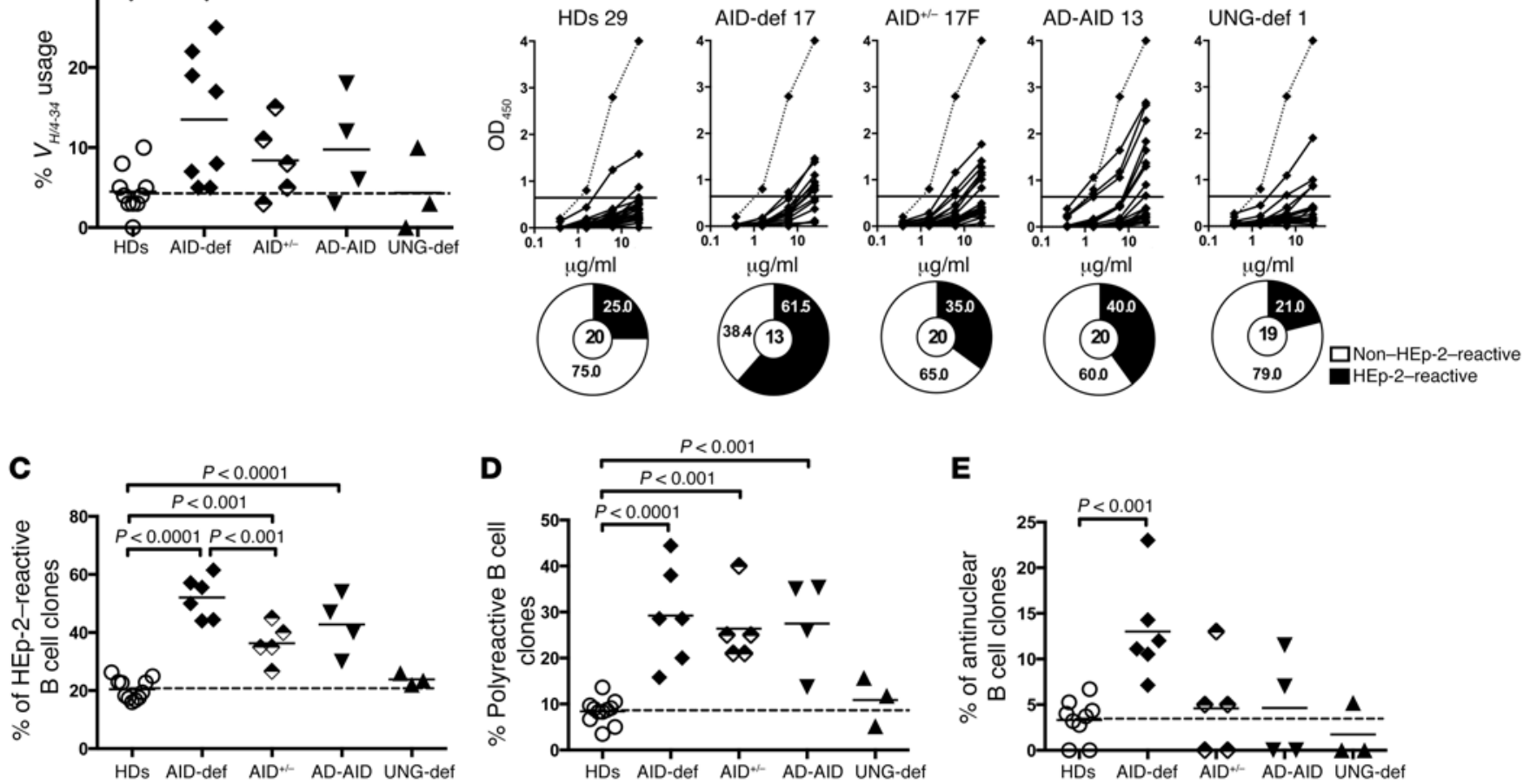

$\mathbf{F}$

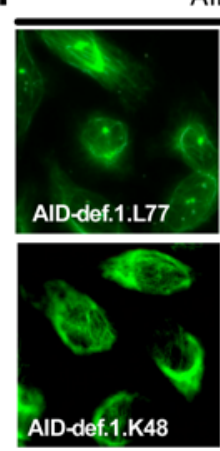

AID-def
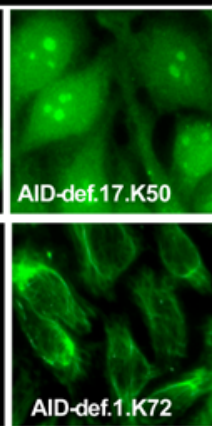

$\mathrm{AlD}^{+/-}$

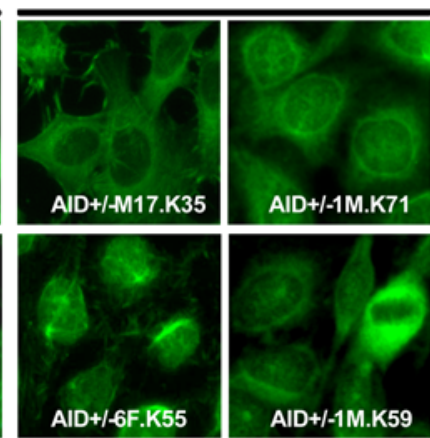

AD-AID

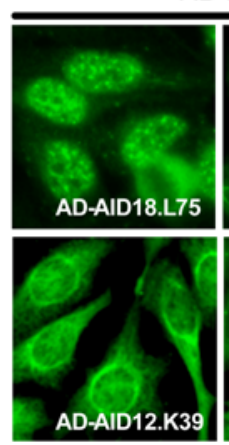

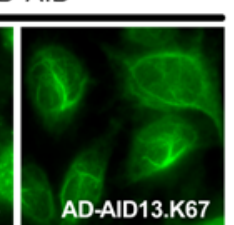

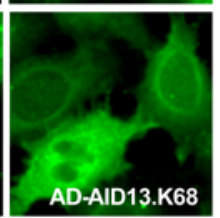

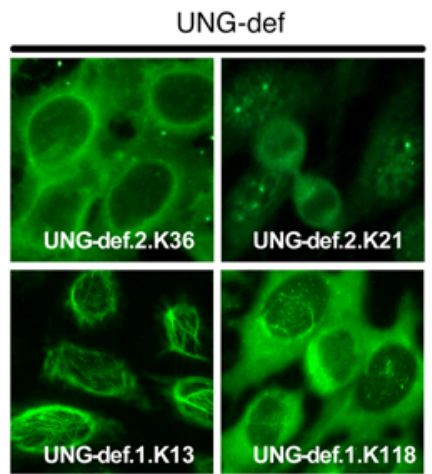

Figure 1. Defective peripheral tolerance checkpoint in patients with AICDA gene mutations. (A) Increased frequency of $V_{H / 4-34}$ gene usage in AID-deficient (AID-def) patients $(n=8)$, asymptomatic healthy heterozygotes ( IID $\left.^{+/-}\right)(n=5)$, and AD-AID patients $(n=4)$ compared with that of HDs $(n=11)$ or UNGdeficient (UNG-def) patients $(n=3)$. Bars indicate the mean \pm SD; dashed line indicates the mean value for the HDs. (B) Antibodies from mature naive $B$ cells were tested by ELISA for anti-HEp-2 cell reactivity. Dotted lines show ED38-positive control, and solid lines show binding for each cloned recombinant antibody. Horizontal lines define the cutoff $\mathrm{OD}_{405}$ for positive reactivity. For each individual, the frequency of autoreactive (black area) and nonautoreactive (white area) clones is summarized in pie charts, with the total number of clones tested indicated in the centers. Summaries of the frequencies of HEp-2-reactive (C), polyreactive (D), and antinuclear (E) mature naive B cells in HDs $(n=11)$ and AID-deficient $(n=6), \operatorname{AID}^{+/-}(n=5)$, AD-AID $(n=4)$, and UNG-deficient $(n=3)$ patients. Bars indicate the mean, and dashed lines indicate the mean value for HDs as a comparison. (F) Diverse staining patterns of antibodies expressed by mature naive B cells obtained by IFA on HEp-2 cells. Original magnification, $\times 40$. $P$ values were determined by ANOVA with Dunnett's correction for multiple comparisons.

an incomplete correlation between serum BAFF concentration and B cell homeostatic expansion (Figure 2C). The dysregulated B cell homeostasis was further evidenced in AID-deficient patients by increased expression of the Ki-67 proliferation marker in mature naive B cells (Supplemental Figure 3, A and B). In addition, in AID-deficient and AD-AID patients, we observed an expansion of unusual $\mathrm{CD} 19^{+} \mathrm{CD} 27^{-} \mathrm{CD} 10^{-} \mathrm{CD} 21^{-/ \mathrm{lo}} \mathrm{B}$ cells that have been reported to contain autoreactive clones and that correlated with increased serum concentrations of IL-21 (Figure 2A and Supplemental Figure 3C) (19). Hence, increased serum BAFF concentrations are associ- ated with the absence of class-switched B cells and do not account for the alteration of the peripheral B cell tolerance checkpoint.

Subjects with AICDA mutation(s) display an altered Treg phenotype and function. Tregs play an important role in preventing the accumulation of autoreactive mature naive B cells, which is revealed by specific defects in the peripheral $\mathrm{B}$ cell tolerance checkpoint in FOXP3-deficient, dedicator of cytokinesis 8-deficient (DOCK8-deficient), and Wiskott-Aldrich patients who have nonfunctional Tregs $(2,18,20)$. In addition, both CD4OL- and AID-deficient patients showed decreased peripheral blood Treg 
A
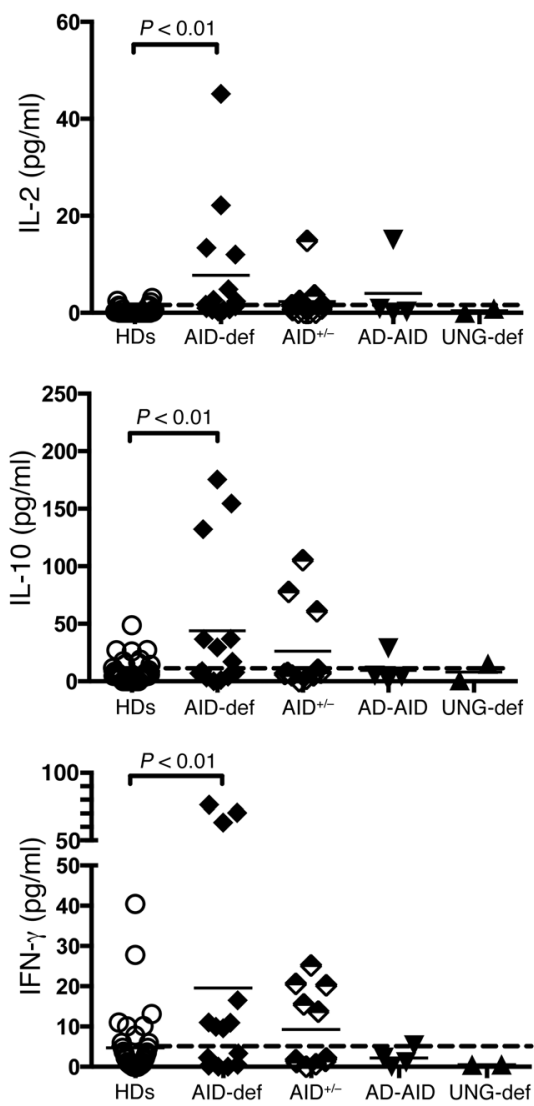

B

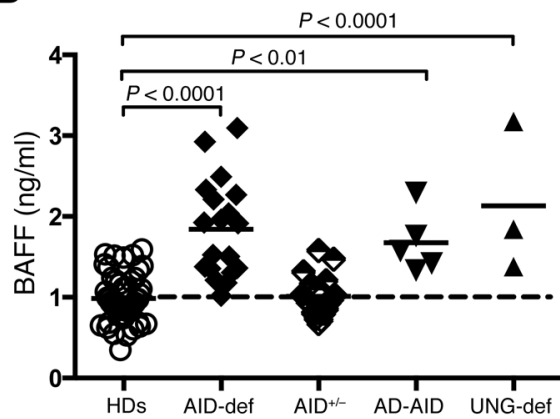

Serum concentration
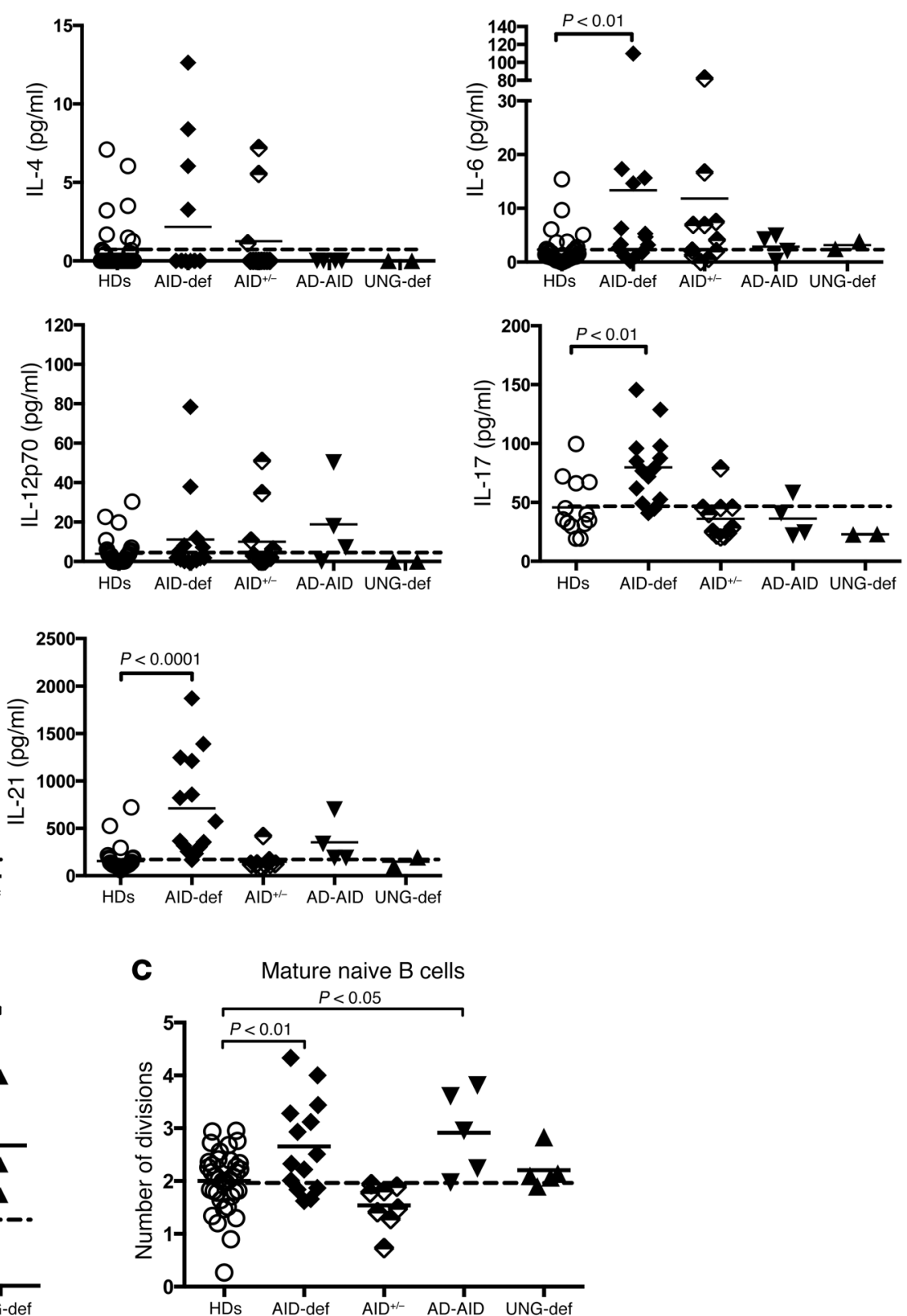

Figure 2. Increased serum cytokine and BAFF concentrations and B cell proliferation in AID-deficient and AD-AID patients. (A) Serum concentrations of various cytokines measured by Luminex or ELISA (pg/ml) in HDs $(n=36)$, AID-deficient patients $(n=14)$, asymptomatic healthy heterozygotes (AID ${ }^{+/}$) $(n=12)$, AD-AID patients $(n=4)$, and UNG-deficient patients $(n=2)$. (B) Serum BAFF concentrations $(\mathrm{pg} / \mathrm{ml})$ in HDs $(n=34)$, AID-deficient patients $(n=17)$, asymptomatic healthy heterozygotes $\left(\operatorname{AID}^{+/-}\right)(n=8)$, and AD-AID $(n=5)$ and UNG-deficient $(n=3)$ patients were measured by ELISA and found to be elevated in all patients with hyper-immunoglobulin M (HICM) syndrome. (C) Evaluation of the number of cell divisions undergone in vivo by KREC analysis of mature naive B cells from HDs $(n=37)$ and AID-deficient $(n=14), \operatorname{AID}^{+-}(n=8), \operatorname{AD}-\operatorname{AID}(n=5)$, and UNG-deficient $(n=5)$ patients. An increased proliferative history was observed in both AID-deficient and AD-AID patients. Bars represent the mean, and dashed lines indicate the mean for HDs as a comparison. $P$ values were determined by ANOVA with Dunnett's correction for multiple comparisons.

frequencies, correlating with an increase in autoreactive mature naive $\mathrm{B}$ cells $(7,8)$. The analysis of additional AID-deficient patients further confirmed that the frequencies of $\mathrm{CD} 4^{+} \mathrm{CD} 25^{\mathrm{hi}}$ $\mathrm{CD}_{127^{\mathrm{l}} \mathrm{FOXP} 3^{+}}$Tregs $(3.5 \% \pm 1.7 \%)$ were decreased in these subjects compared with frequencies detected in HDs $(5.1 \% \pm$ $1.7 \%, P<0.01$ ) (Figure 3, A and B). In contrast, $\mathrm{AID}^{+/}$individuals, AD-AID patients, and UNG-deficient patients displayed normal Treg frequencies in their blood (Figure 3, A and B). In addition,
Tregs from AID-deficient patients exhibited an abnormal phenotype evidenced by a significant increase in the Ki-67 proliferative marker, suggesting defective suppressive activity (Figure 3C) (20). A direct assessment of the in vitro suppressive capacity of Tregs showed that Tregs from AID-deficient patients, AID ${ }^{+/-}$heterozygotes, and AD-AID patients had an impaired ability to inhibit the proliferation of CFSE-labeled $\mathrm{CD} 3^{+} \mathrm{CD} 4^{+} \mathrm{CD} 25^{-}$autologous and heterologous $\mathrm{T}$ responder (Tresp) cells (Figure 4, A-C). In con- 
A

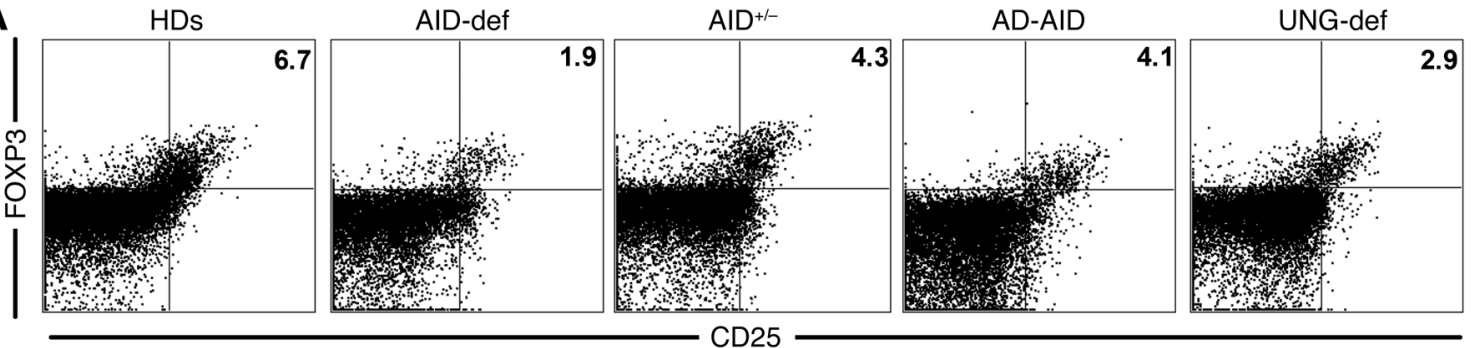

B
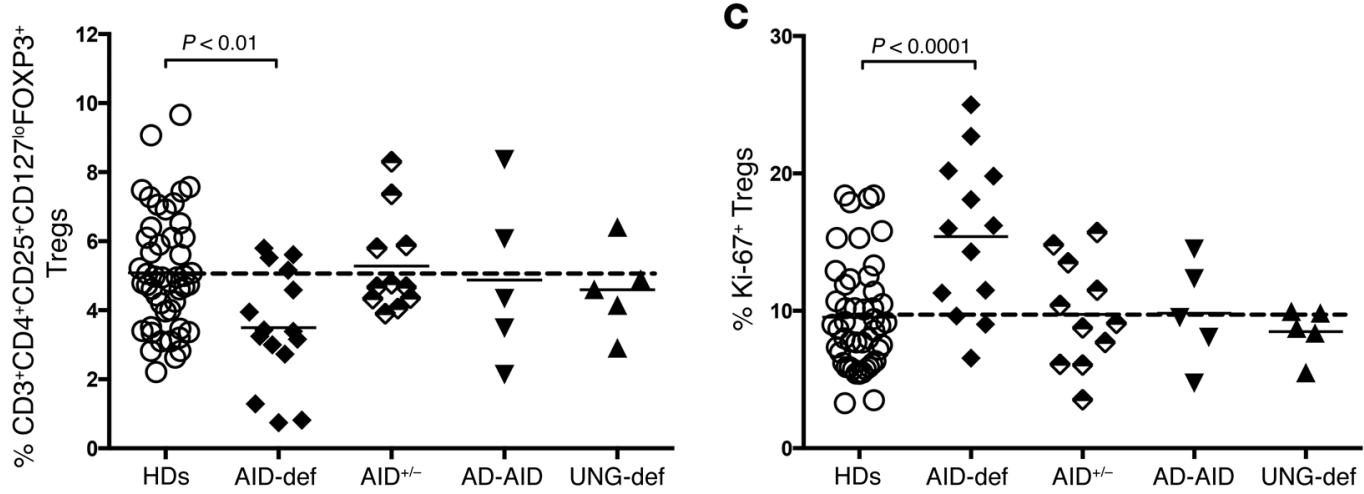

Figure 3. Altered Treg phenotype is restricted to AID-deficient patients. (A) Representative CD25 and FOXP3 staining on CD3 $3^{+} \mathrm{CD} 4^{+}$cells. (B and C) Summary of the data shows lower frequencies of $\mathrm{CD} 3^{+} \mathrm{CD} 4^{+} \mathrm{CD} 25^{+} \mathrm{CD} 127^{\circ} \mathrm{FOXP3}{ }^{+}$Tregs but a higher proportion of Ki-67 ${ }^{+}$Tregs in AID-deficient patients $(n=13)$ compared with frequencies detected in $\mathrm{HDs}(n=48)$ and $\operatorname{AID}^{+/-}(n=11)$, AD-AID $(n=5)$, and UNG-deficient $(n=5)$ patients. Bars represent the mean, and dashed lines indicate the mean for HDs as a comparison. $P$ values were determined by ANOVA with Dunnett's correction for multiple comparisons.

trast, Tregs from UNG-deficient patients showed normal in vitro suppression, which correlated with their low frequencies of autoreactive mature naive B cells (Figure $4, \mathrm{~A}^{-} \mathrm{C}$ ). In addition, Tresps from AID-deficient patients were refractory to suppression by HD Tregs, whereas the proliferation of Tresps from asymptomatic healthy heterozygotes as well as AD-AID and UNG-deficient patients could be suppressed by Tregs from HDs (Figure 4D). In conclusion, impaired Treg suppressive function correlates with peripheral B cell tolerance checkpoint defects in $\mathrm{AID}^{+/}$individuals as well as AD-AID and AID-deficient patients who also have $\mathrm{T}$ cells that are refractory to Treg suppression.

Defective Treg suppressive function is associated with increased cytokine production of Tresps. We have previously reported that increased Tresp proliferation associated with decreased Treg suppression could result from the dysregulated production of specific cytokines (15). We therefore analyzed cytokine production ex vivo by $\mathrm{CD} 4^{+} \mathrm{T}$ cells freshly isolated from AID-deficient patients or $\mathrm{AID}^{+/-}$heterozygotes. We could not assess cytokine production in AD-AID or UNG-deficient patients, because the results are severely altered when frozen peripheral blood mononuclear cells (PBMCs) are used. We observed an increase in IL-4 production by $\mathrm{CD}^{+} \mathrm{T}$ cells from subjects with AICDA mutation(s), which have previously been reported to interfere with proper Treg function $(15,21)$, whereas IL-10 production did not appear to be dysregulated (Figure 5, A and B). We also found an increase in the production of IL-2, IL-21, and IFN- $\gamma$ by CD $4^{+}$T cells from AID-deficient patients, further attesting to the hyperresponsiveness of these cells (Figure 5, A and B). Quantitative PCR analyses validated the increased production of IL-2, IL-4, and IL-21 by freshly iso- lated $\mathrm{CD} 4^{+} \mathrm{T}$ cells from AID-deficient patients and in some from $\mathrm{AID}^{+/-}$subjects (Supplemental Figure 4). Several of these cytokines induce an inability of Treg cells to suppress Tresps, similar to what we observed in cocultures of Tregs and Tresps isolated from AID-deficient patients (Supplemental Figure 5, A and B) (15). IL-12, which we found to be increased in the serum of some AD-AID patients (Figure 2A), has also been shown to interfere with proper Treg function (22). In addition, we directly tested the effect of cytokines solely on Treg function by preincubating HD Tregs for 4 days with various ILs and then assessed their suppressive ability on fresh Tresps in the absence of any additional cytokines. We found that Tregs preincubated with IL-4 and IL-12 were substantially less effective at inhibiting Tresp proliferation than were nontreated Tregs, whereas preincubation with IL-10 and IL-21 did not affect Treg function (Figure 5, C and D). In contrast, Tregs cultured with elevated IL-2 concentrations showed an increased capacity to inhibit Tresp proliferation as reported previously (23) (Figure 5D). When HD Tregs were preincubated with all the cytokines (referred to as "mix" in the figure) found to be elevated in AID-deficient patients, we observed an inability of these Tregs to suppress Tresp proliferation (Figure 5D). Altogether, increased cytokine production in patients with AICDA mutation(s) may contribute to the poor Treg suppressive function and a defective peripheral B cell tolerance checkpoint in these individuals.

Increased circulating Tfh cells in individuals with AICDA gene mutation(s). To assess whether peripheral tolerance was further breached in individuals with AICDA or UNG gene mutations, we analyzed the presence of autoantibodies and antinuclear antibodies (ANAs) in the plasma or serum of these CSR-D patients and 
A
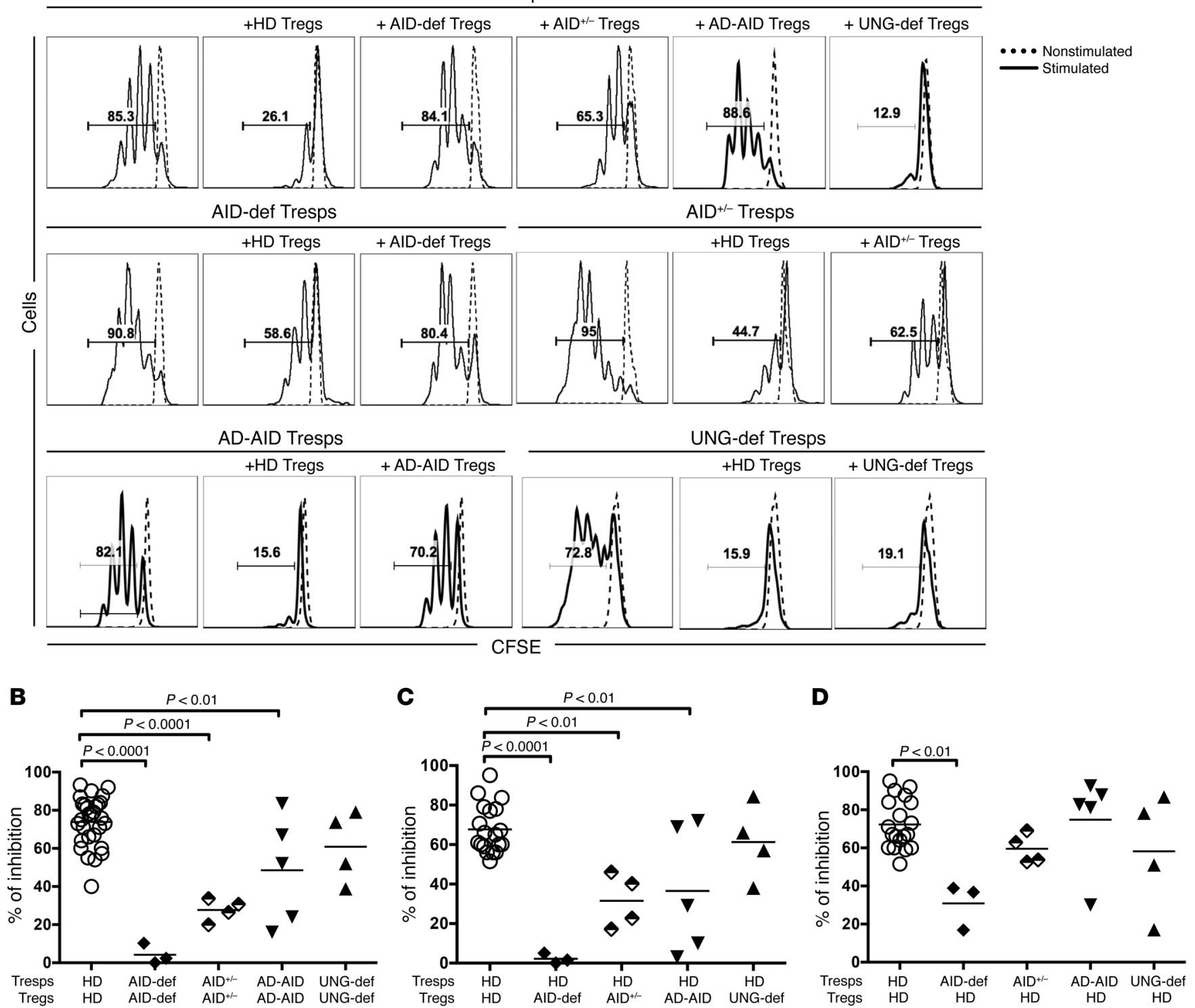

Figure 4. Peripheral B cell tolerance defects in patients carrying AICDA mutations correlate with impaired Treg function. (A) Representative histograms of Treg-mediated suppression of autologous and heterologous CFSE-labeled Tresps on day 3.5 from 1 AID-deficient patient, 1 asymptomatic healthy carrier, 1 AD-AID patient, and 1 UNG-deficient patient compared with those from a HD. Dashed line shows nonstimulated Tresps. (B) Autologous suppressive capacity of Tregs from HDs $(n=19)$, AID-deficient patients $(n=3)$, AID ${ }^{+-}$subjects $(n=4)$, AD-AID patients $(n=5)$, and UNG-deficient patients $(n=4)$. Bars indicate the mean, whereas $\mathbf{C}$ and $\mathbf{D}$ display the heterologous suppressive capacity of mixed Treg/Tresp cocultures. $P$ values were determined by ANOVA with Dunnett's correction for multiple comparisons.

$\mathrm{AID}^{+/-}$individuals. We first confirmed, by analyzing additional AID-deficient patients, that all 14 of these patients had high titers (1:320) of IgM autoantibodies and ANAs in their plasma/serum (Figure 6A) (8). In contrast, no serum IgM autoantibodies could be detected in asymptomatic $\mathrm{AID}^{+/-}$heterozygotes or in $\mathrm{AD}-\mathrm{AID}$ or UNG-deficient patients (Figure 6A). Since T cell help is important for ANA production, we analyzed circulating CXCR5 $5^{+} \mathrm{PD}-1^{+} \mathrm{T}$ follicular helper ( $\mathrm{Tfh}$ ) cells that are efficient at inducing antibody secretion $(16,24,25)$. We found the highest frequencies of $\mathrm{CD}^{+}$ $\mathrm{CD}^{+}{ }^{+} \mathrm{CXCR}^{+} \mathrm{PD}-1^{+} \mathrm{Tfh}$ cells in AID-deficient patients $(7.2 \% \pm$ $2.6 \%, P<0.0001)$ and an increase in Tfh cells in AD-AID patients $(4.8 \% \pm 1.5 \%, P<0.01)$ compared with HDs $(1.8 \% \pm 1.4 \%)$ (Figure
6, B and C). Asymptomatic healthy heterozygotes also showed a modest increase in Tfh cells $(3.1 \% \pm 1.6 \%)$, although differences with HDs did not reach statistical significance when analyzed by ANOVA with Dunnett's correction for multiple comparisons (Figure $6, \mathrm{~B}$ and $\mathrm{C})$. In contrast, UNG-deficient patients showed no increase in Tfh cells (2.2\% $\pm 0.7 \%)$ (Figure 6, B and C). Circulating Tfh cells in AID-deficient patients resembled tonsillar counterparts because of increased ICOS expression (Figure 6, B and C). Tfh cells were most prevalent in the blood of patients with the lowest frequencies of Tregs (Figure 6D), an inverse correlation previously observed in CVID patients (16). Hence, AICDA mutations correlate with elevated frequencies of circulating Tfh cells that 
A
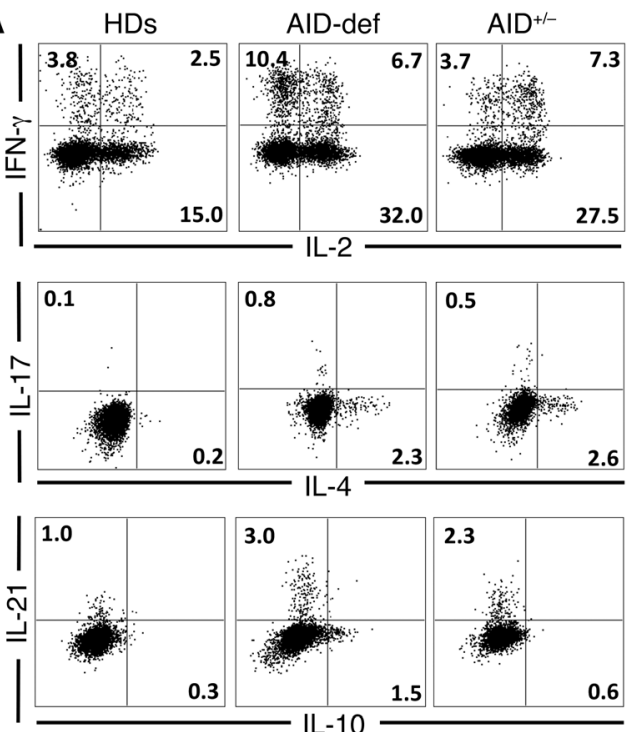

B
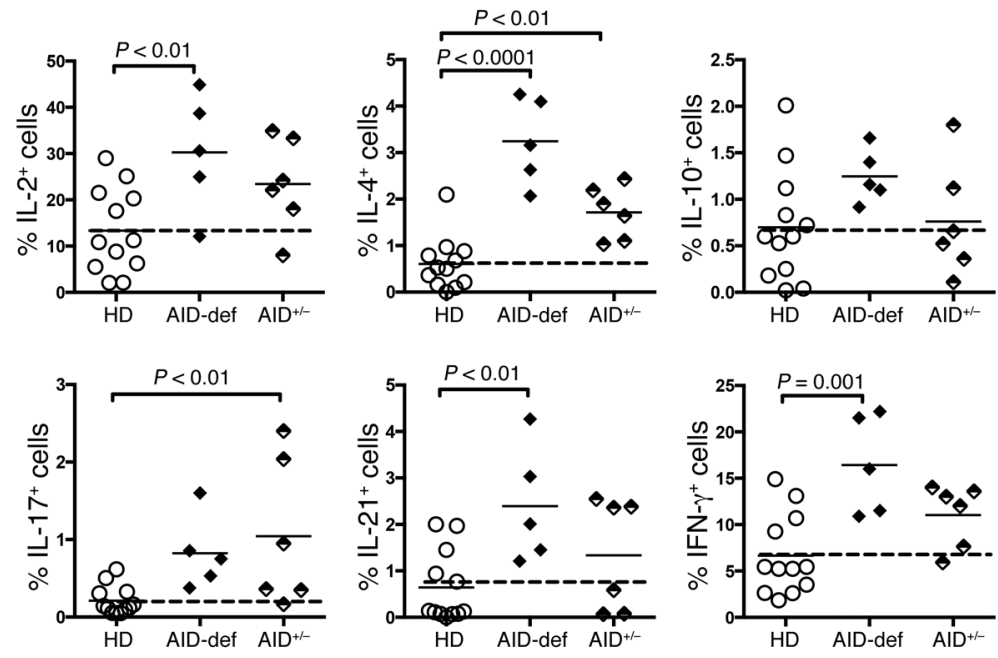

C

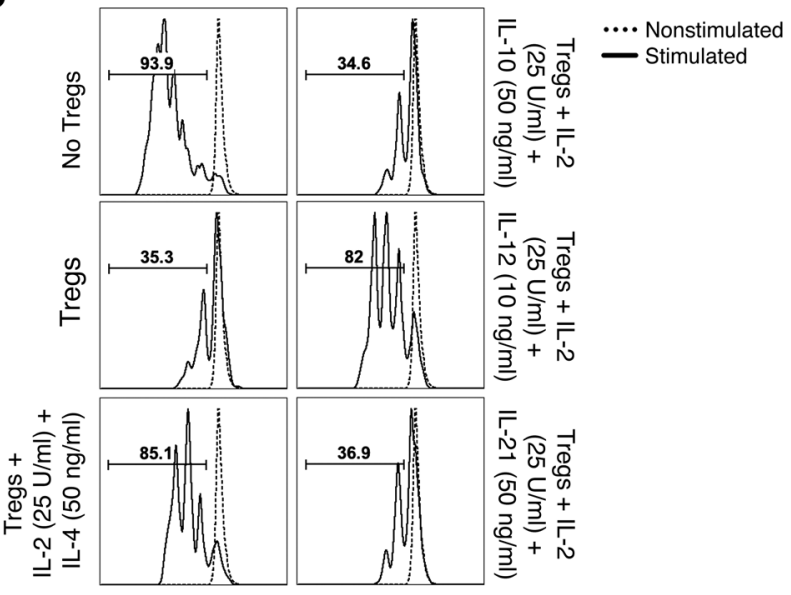

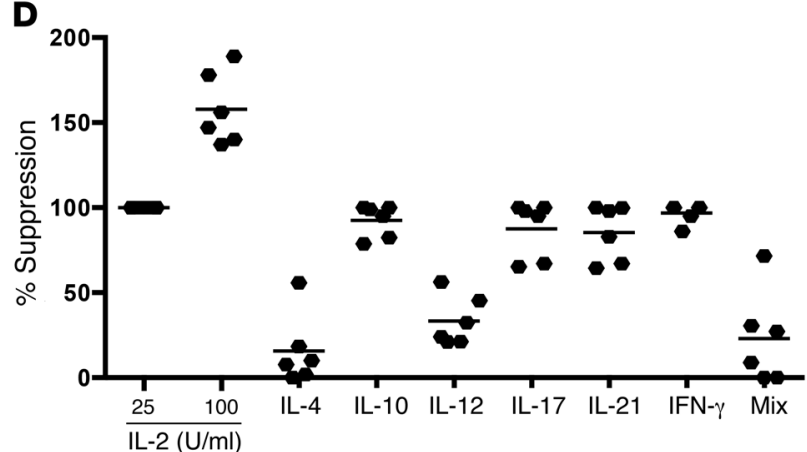

Figure 5. Increased cytokine production by Tresps interferes with proper Treg suppression in all individuals carrying AICDA mutations. (A) Representa-

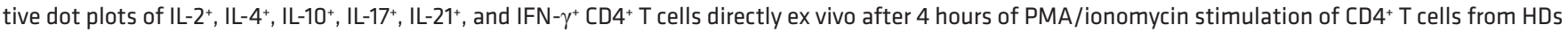
$(n=12)$, AID-deficient patients $(n=5)$, and AID ${ }^{+/-}$patients $(n=6)$. Data are summarized in B, where bars indicate the mean and dashed lines the mean for HDs. $P$ values in B were determined by ANOVA with Dunnett's correction for multiple comparisons. (C) Representative histograms of Tregs preincubated 4 days with or without the addition of IL-2, IL-4, IL-10, IL-12, or IL-21 prior to coculture with freshly isolated autologous, CFSE-labeled Tresps. Dashed lines show nonstimulated Tresps. Data are summarized in $\mathbf{D}(n=6)$.

express ICOS and may lead to a breach in B cell tolerance and the production of serum IgM autoantibodies in AID-deficient patients who display the highest elevation in Tfh cell numbers.

Altered SHM in subjects with AICDA mutation(s) correlates with their defective peripheral $B$ cell tolerance checkpoint. The lack of CSR involvement in establishing the peripheral B cell tolerance checkpoint suggested a potential role for SHM in the regulation of this selection step. However, examination of IgM $V_{H / 5-51}$ in total CD27 $7^{+}$ B cells and $V_{H / 3-23}$ rearrangements in purified $\mathrm{IgM}^{+} \mathrm{CD} 27^{+}$memory $\mathrm{B}$ cells revealed normal SHM frequencies in asymptomatic $\mathrm{AID}^{+/-}$ individuals and AD-AID patients (10). This observation contrasted with the decreased induction of SHM in B cells from $\mathrm{AID}^{+/}$mice, in which AID expression was decreased compared with that observed in their WT counterparts $(26,27)$. Since we recently observed a similar decrease in expression of AID protein in EBV cell lines and activated B cells from $\mathrm{AID}^{+/-}$individuals (28), we assessed the incidence of SHM in AD-AID patients and $\mathrm{AID}^{+/-}$subjects by analyzing heavy chain $(I G H)$ gene sequences amplified from single $\mathrm{CD} 27^{+} \mathrm{IgM}^{+}$and $\mathrm{CD} 27^{+} \mathrm{IgG}{ }^{+}$memory B cells and compared them with the sequences amplified from $B$ cell counterparts from $H D s$ as well as AID-deficient and UNG-deficient patients. We found that $\mathrm{CD} 27^{+} \mathrm{IgM}^{+} \mathrm{B}$ cells from HDs showed, on average, $10.8 \pm 6.8$ mutations in their IGH genes, as previously reported for different donor controls (Figure 7A) (29). CD27+IgM+ B cells from $\mathrm{AID}^{+/}$individuals and a UNG-deficient patient harbored mutation numbers that were similar to those in $\mathrm{HDs}$, whereas $\mathrm{CD} 27^{+} \mathrm{IgM}^{+} \mathrm{B}$ cells from AID-deficient patients were devoid of SHM, as expected (Figure $7 \mathrm{~A})$. In contrast, $\mathrm{CD} 27^{+} \mathrm{IgM}^{+} \mathrm{B}$ cells from AD-AID patients showed decreased SHM frequencies, with only $3.9 \pm 4.3$ mutations, on average, in $I G H$ genes (Figure $7 \mathrm{~A}$ ). $\mathrm{CD}^{2} 7^{+} \mathrm{Ig} \mathrm{G}^{+}$memory B cells from $\mathrm{AID}^{+/}$subjects displayed significantly decreased SHM in their IgH genes, with the average only reaching $16.5 \pm 7.8$ mutations com- 
A

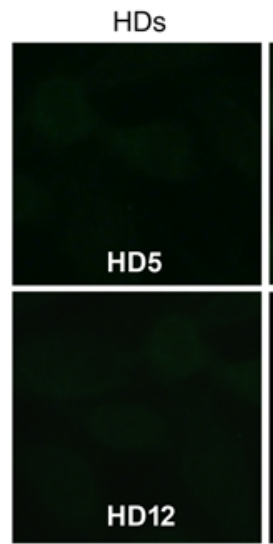

SLE

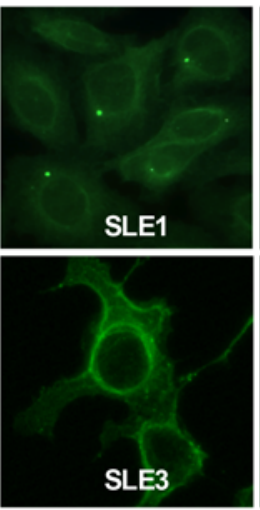

AID-def

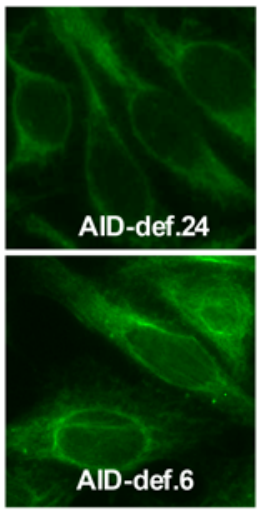

$\mathrm{AID}^{+/-}$

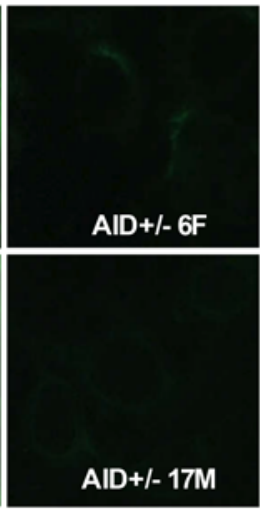

AD-AID

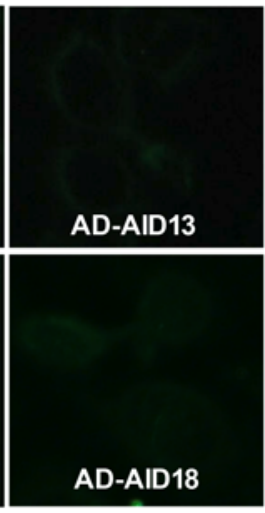

$0 / 4$
UNG-def

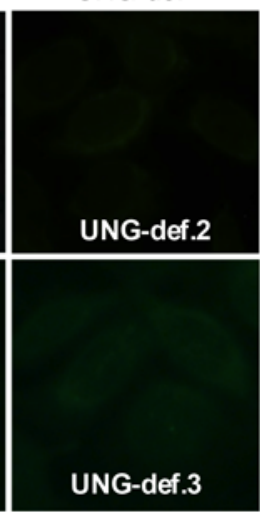

$0 / 3$
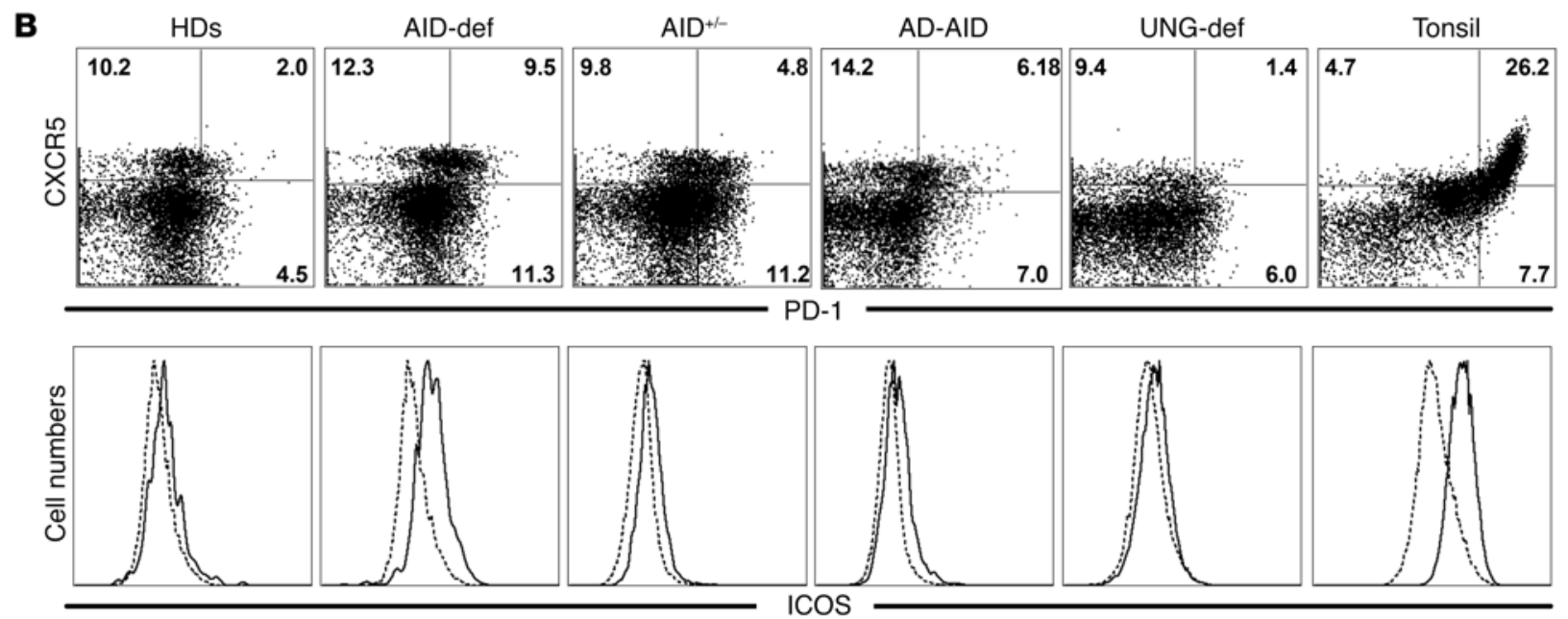

--- $\mathrm{CD}^{+}{ }^{+} \mathrm{CD} 4{ }^{+} \mathrm{CXCR} 5-\mathrm{PD} 1$ $-\mathrm{CD} 3{ }^{*} \mathrm{CD} 4{ }^{+} \mathrm{CXCR} 5{ }^{+} \mathrm{PD} 1{ }^{+}$
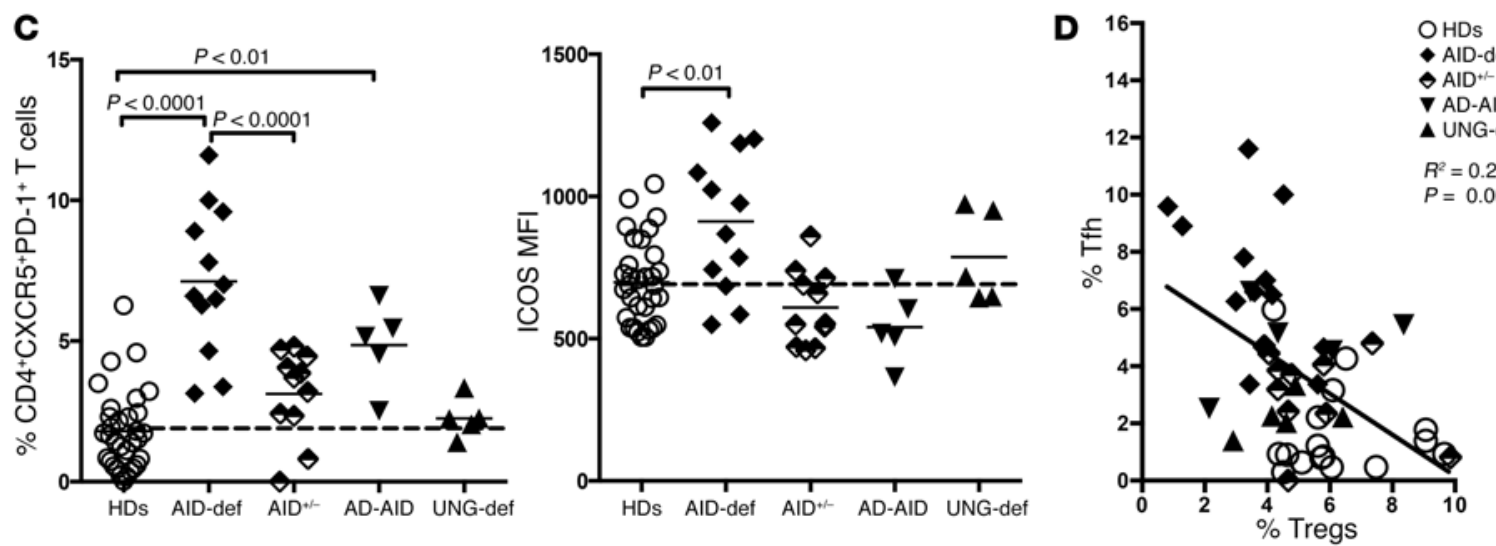

Figure 6. B cell tolerance is further breached in AID-deficient patients and correlates with the presence of circulating Tfh cells. (A) IgM autoantibodies and ANAs with diverse staining patterns were only present in SLE and AID-deficient patients. Serum dilution: 1:80. (B) Representative dot plots of PD-1 and CXCR5 staining on CD3 ${ }^{+} \mathrm{CD} 4^{+}$cells and histograms of ICOS expression on CD3 ${ }^{+}$CD4 ${ }^{+}$CXCR5 $-P D-1^{-}$T cells (dashed line) compared with $C D 3^{+}$CD4 ${ }^{+}$ CXCR5 ${ }^{+} P D-1^{+} T$ cells (solid line) in all groups. (C) Summary of data comparing the frequency of circulating CD3 ${ }^{+}$CD $4^{+}$CXCR5 $5^{+} P D-1^{+}$cells and the mean fluorescence intensity (MFI) of ICOS in HDs $(n=32)$ versus AID-deficient patients $(n=11)$, AID ${ }^{+/}$subjects $(n=10)$, AD-AID patients $(n=5)$, and UNGdeficient patients $(n=5)$. Bars indicate the mean; dashed line indicates the mean for HDs as a comparison. (D) The frequency of circulating Tfh cells inversely correlated with the frequency of circulating Tregs in all subjects. Line was obtained by linear regression analysis. $P$ values were determined by ANOVA with Dunnett's correction for multiple comparisons. 
A

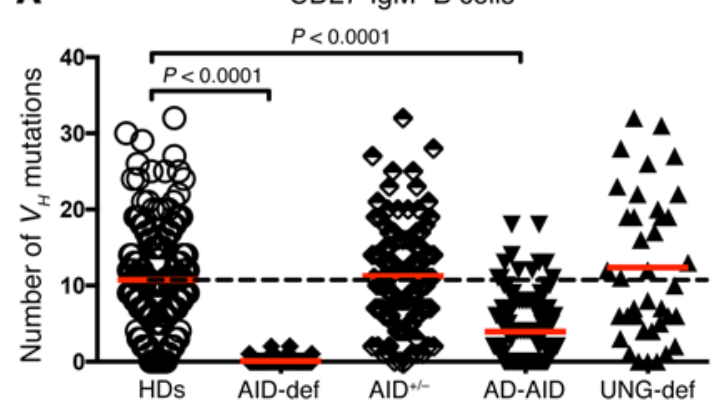

C
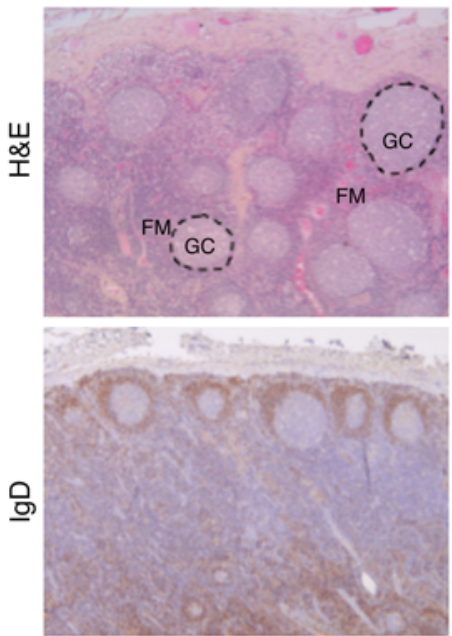
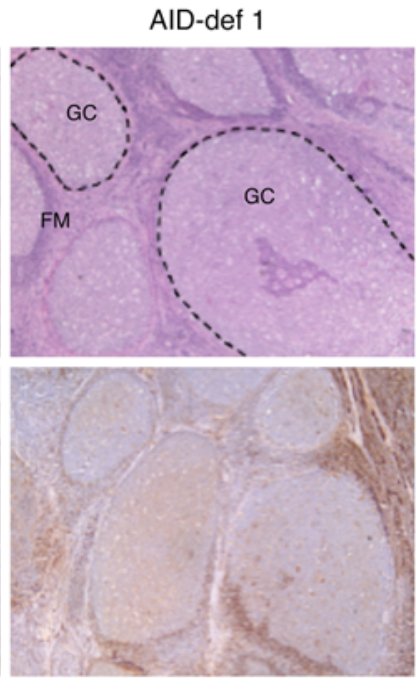

B

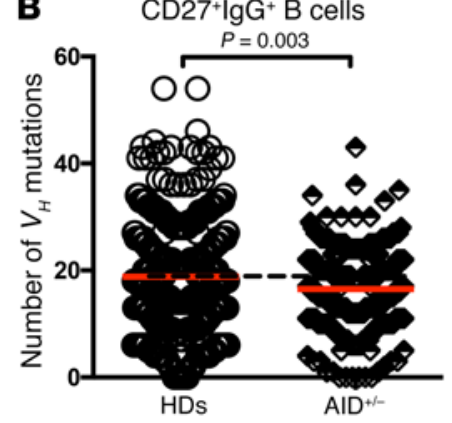

UNG-def 3

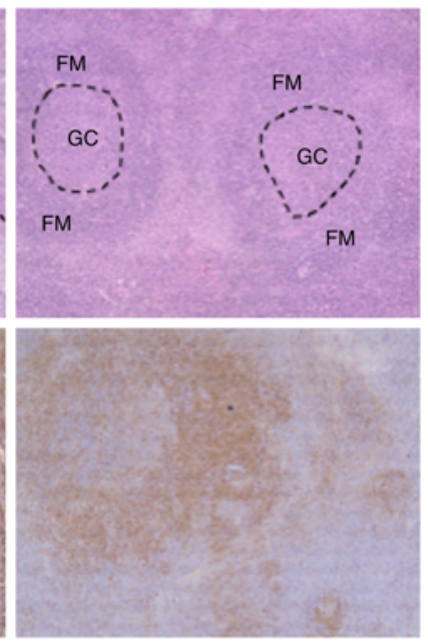

Figure 7. Decreased SHM frequencies in memory B cells from subjects with AICDA gene mutation(s). (A) Decreased SHM in IgM+ memory B cells from AD-AID patients. The number of mutations evaluated in $V_{H}$ sequences derived from single CD27+IgM+ B cells from HDs $(n=16)$, AID-deficient patients $(n=5)$, AID ${ }^{+/-}$subjects $(n=4)$, AD-AID patients $(n=4)$, and 1 UNG-deficient patient is shown. $P$ values were determined by ANOVA with Dunnett's correction for multiple comparisons. (B) Decreased SHM in IgG+ memory B cells from $\mathrm{AlD}^{+/-}$subjects. The number of mutations in $V_{H}$ sequences from single $C D 27^{+} I g \mathrm{CG}^{+} \mathrm{B}$ cells from $\mathrm{HDs}(n=15)$ and $\mathrm{AID}^{+/-}$healthy subjects $(n=7)$ is shown. Each sequence is depicted by a symbol, and bars represent the mean. $P$ values were determined by an unpaired, 2-tailed Student's $t$ test. (C) Left: Control reactive cervical lymph node with lymphoid follicles composed of normal-sized GCs and a well-defined follicular mantle (FM). Middle: cervical lymph node biopsies from AID-deficient patient 1 showing lymphoid follicular hyperplasia with giant GCs and a characteristic reduced FM. Right: cervical lymph node biopsy from UNG-deficient patient 3 with lymphoid follicle hyperplasia with ill-defined GC separation from the normal-sized mantle zone. Original magnification, $\times 50$.

pared with $18.8 \pm 9.8$ mutations in the $I g H$ genes of HDs $(P=0.003$, Figure 7B) (30). Surprisingly, IgG antibodies encoded by $V_{H / 3-23}$ genes did not follow this trend and displayed similar SHM averages between HDs and $\mathrm{AID}^{+/}$heterozygotes, as previously reported for mutated IgM sequences in these subjects (Supplemental Figure 6) (10). Finally, lack of SHM in AID-deficient patients was found to be associated with enlarged germinal centers (GCs) (Figure 7C) (31). We could not assess GCs from asymptomatic $\mathrm{AID}^{+/-}$subjects or AD-AID patients, but we managed to obtain a lymph node biopsy from a UNG-deficient patient that revealed normal-sized GCs, a finding consistent with the normal SHM responses identified in the $\mathrm{CD}^{2} 7^{+} \mathrm{IgM}^{+}$memory B cells (Figure 7A). However, the morphology of GCs from the UNG-deficient patient was not completely normal; GCs showed ill-defined borders and appeared to include some IgD $\mathrm{I}^{+} \mathrm{B}$ cells (Figure 7C and data not shown). We conclude that both $\mathrm{AID}^{+/}$individuals and AD-AID patients show decreased $\mathrm{SHM}$ in $\mathrm{CD} 27^{+} \mathrm{IgG}^{+}$and $\mathrm{CD} 27^{+} \mathrm{IgM}^{+} \mathrm{B}$ cells, respectively, suggestinto the periphery $(8,28,32)$. However, we previously reported that autoreactive clones can be prevented from colonizing the mature naive B cell compartment when Tregs are functional, despite the impaired removal of developing autoreactive immature B cells in the bone marrow (16). In addition, AD-AID patients show only a defective peripheral B cell tolerance checkpoint, since their central $\mathrm{B}$ cell tolerance was found to be functional, thereby excluding a contribution from the bone marrow to peripheral selection impediments in subjects with AICDA gene mutation(s) (28). For those reasons, we sought to determine which factors control the peripheral B cell tolerance checkpoint that is defective when SHM is impaired.

Decreased Treg numbers or function and elevated BAFF concentrations have both been reported to be associated with a dysregulated peripheral B cell tolerance checkpoint $(2,14,16,18,20)$. Elevated serum BAFF concentrations can potentially impact the peripheral checkpoint due to the ability of BAFF to rescue autoreactive $B$ cells from cell death in mice $(33,34)$. However, the 


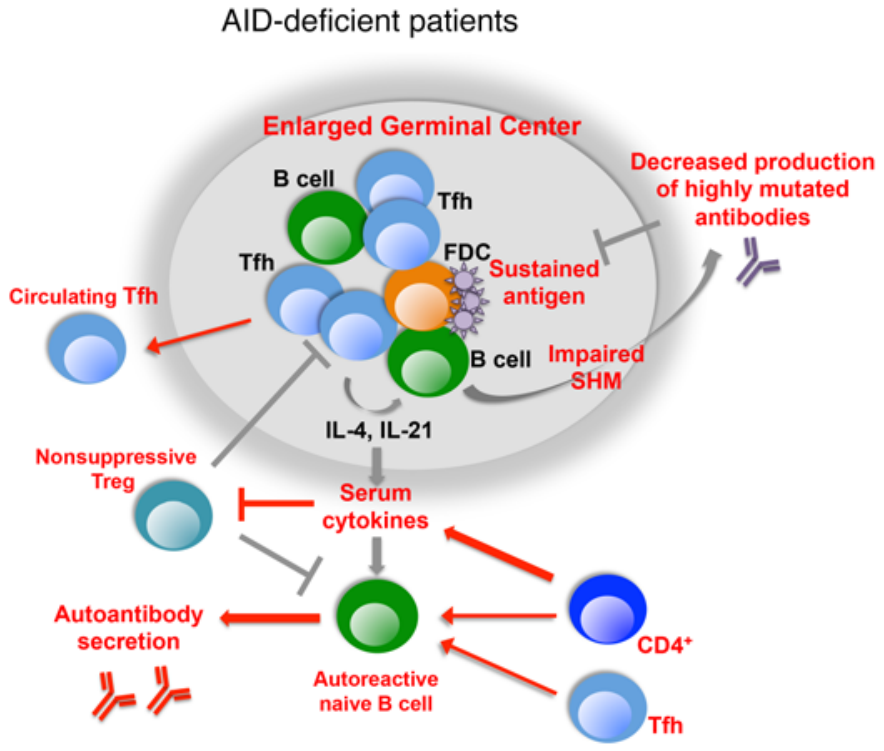

Figure 8. Disruption of the peripheral B cell tolerance checkpoint by untamed GC reactions. Decreased SHM processes result in a reduced efficiency at generating highly mutated specific antibodies, potentially leading to enhanced GC reactions, increased Tfh cell production, and cytokine secretion. These cytokines interfere with Treg suppressive function and potentially result in a defective peripheral B cell tolerance checkpoint. This impaired selection step allows the accumulation of large numbers of autoreactive B cells in the periphery that could be activated by Tfh cells and systemic cytokines that support not only autoantibody secretion but potentially the development of autoimmune manifestations.

impaired peripheral $\mathrm{B}$ cell tolerance checkpoint in asymptomatic $\mathrm{AID}^{+-}$individuals as well as in FOXP3-, SAP-, and DOCK8deficient patients, all of whom display normal serum BAFF concentrations, suggests that the regulation of this checkpoint is independent of $\operatorname{BAFF}(2,15)$. A functional peripheral B cell tolerance checkpoint in UNG-deficient patients further reveals that a 2- to 3 -fold elevation in serum BAFF concentration does not induce defects in peripheral B cell selection. Hence, anti-BAFF therapy, which has shown some efficacy in systemic lupus erythematosus (SLE), may not improve the condition of these patients by restoring their impaired counterselection of autoreactive B cells (35-37). The elevated serum BAFF concentrations in patients with CSR-D as well as in many CVID patients correlate, rather, with decreased memory B cell numbers $(16,38,39)$. Interestingly, many patients with autoimmune diseases such as SLE or rheumatoid arthritis who show elevated serum BAFF concentrations have been reported to have decreased circulating memory B cells in their blood (40-43). Compared with naive B cells, memory B cells that express increased cell-surface transmembrane activator and CAML interactor (TACI), another BAFF receptor, may constitute a sink for serum BAFF in humans. In agreement with this hypothesis is the absence of elevated serum BAFF concentrations in Aicda-KO mice, which correlates with the very low proportion of memory $\mathrm{B}$ cells among total B cells in mice compared with humans $(32,44)$.

Circulating mature naive B cells contain many autoreactive clones in FOXP3-deficient patients who lack functional Tregs, suggesting a role for Tregs in controlling the peripheral B cell tolerance checkpoint (2). Moreover, the accumulation of autoreactive mature naive B cells in DOCK8-deficient, Wiskott-Aldrich, and CVID patients correlates with abnormal Treg suppressive function $(2,16,18,20)$. Our current finding of impaired Treg suppres- sive function in individuals with AICDA mutation(s) who show defective peripheral $B$ cell selection further supports a major role for Tregs in regulating this checkpoint. How can AICDA mutations affect Treg function? Since T cells do not express AID, the defective suppressive ability of Tregs from AID-deficient patients and asymptomatic $\mathrm{AID}^{+/-}$subjects should be the consequence of extrinsic dysregulated factors. In line with this hypothesis is the overexpression of many cytokines such as IL- 4 and IL- 21 by CD $4^{+}$ T cells from subjects with AICDA mutation(s) that inhibit Treg suppressive function (15). Other cytokines such as IL-6 and IL-12 produced by other cell types and known to alter Treg suppression were also elevated in the serum of individuals carrying recessive and dominant $A I C D A$ mutations, respectively $(15,22)$. In addition, we discovered that, of the cytokines suspected to alter Treg suppression, IL-12 and IL-4, which are key cytokines for Th1 and Th2 responses, respectively, had inhibitory effects on Treg function upon pre-exposure. We hypothesize that IL-12 and IL-4 secretions during Th1, Th2, and Tfh responses not only polarize T cell phenotype and function but also inhibit Tregs, thereby allowing autoimmune responses to proceed. Moreover, $\mathrm{CD} 4^{+} \mathrm{T}$ cells from AIDdeficient patients were refractory to Treg suppression, a feature also identified in SAP-deficient patients who also had hyperactive $\mathrm{CD}^{+} \mathrm{T}$ cells secreting many cytokines and a defective peripheral B cell tolerance checkpoint (15).

The altered $\mathrm{CD} 4^{+} \mathrm{T}$ cell compartment in subjects with AICDA mutation(s) also included an increase in circulating Tfh cells; Tfh cells, in conjunction with elevated serum IL-21 and other cytokines, in AID-deficient patients may allow the activation of autoreactive $B$ cells, leading to the secretion of unmutated ANAs (45, 46). A similar scenario was observed in many CVID patients who had increased frequencies of Tfh cells, together with enhanced 
production of IL-4 production and serum ANAs, all of which correlated with decreased Treg frequencies, an impaired peripheral B cell tolerance checkpoint, and autoimmune manifestations (16). In contrast, $\mathrm{AID}^{+/-}$heterozygotes and $\mathrm{AD}$-AID patients who show some AID activity had a milder phenotype than did AID-deficient patients, as illustrated by an absence of serum ANAs, suggesting that peripheral tolerance is not breached, despite an abnormal peripheral B cell tolerance checkpoint. These observations are not paradoxical, because it is unlikely that autoreactive mature naive $\mathrm{B}$ cells from $\mathrm{AID}^{+/-}$heterozygotes and AD-AID patients secrete the antibodies expressed on their cell surface in the absence of $\mathrm{T}$ cell help, since these individuals only showed a modest increase in Tfh cells and serum cytokines that may not be sufficient to overrule other more downstream checkpoints preventing the activation of these self-reactive clones and production of autoantibodies. In line with this hypothesis, none of these individuals has been reported to suffer from autoimmune conditions. Hence, elevated Tfh cells with increased ICOS expression and serum cytokines, in combination with decreased Treg frequency and function, may favor autoreactive $\mathrm{B}$ cell activation and ANA production.

How do AICDA mutation(s) result in increased circulating Tfh cells? It has previously been reported that AID deficiency results in enlarged GCs in both mice and humans $(5,6)$. Tfh cells are normally found in GCs, where they activate B cells through cell-cell interactions and production of cytokines including IL-4 and IL-21 (47). Tfh cells induce AID expression in GC B cells, leading to the initiation of SHM that ultimately increases antibody affinity through antigen selection during immune responses (48-50). The production of mutated high-affinity antibodies was recently shown to downregulate GC reactions, probably by eliminating specific antigens on follicular DCs (FDCs) (51). The presence of high-affinity IgG ${ }^{+}$memory $B$ cells that can rapidly differentiate into plasma cells secreting large amounts of specific antibodies also inhibits GC formation, further suggesting that high-affinity antibodies generated by SHM play an important role in controlling GC development (52). A failure to induce SHM, and probably to generate high-affinity-specific antibodies in AID-deficient patients, may therefore lead to sustained antigen expression on FDCs in GCs, leading to prolonged $\mathrm{B}$ and $\mathrm{T}$ cell activation and proliferation as well as to increased production of Tfh cells and secretion of their cytokines, namely IL-4, IL-10, and IL-21, which can all be found in the blood of AID-deficient patients $(24,25)$ (Figure 8). In addition, increased resistance to apoptosis in AID-deficient B cells may further support an enhanced GC reaction $(32,53)$. This model is corroborated by observations in mice carrying a knockin point mutation in AICDA, which decreases the SHM activity of AID without altering CSR function (54). GC B cell hyperplasia in gut lymphoid tissue was observed in these mice and disappeared after antibiotic treatment, similar to what was observed in Aicda ${ }^{-/-}$mice (55). Hence, loss of SHM independently of CSR defects leads to increased GC formation associated with the inability to control microbiota. This scenario may also apply to both $\mathrm{AID}^{+/-}$subjects and AD-AID patients, because these subjects showed decreased SHM in their $\operatorname{IgG}^{+}$and $\operatorname{IgM}^{+}$memory B cells, respectively. Altered SHM processes in these individuals had initially been missed because of the small numbers of mutated sequences obtained from the amplification of $V_{H / 5-51}$ and $V_{H / 3-23}$ genes (10). However, our more global and unbiased approach revealed lower SHM frequencies in single $\mathrm{IgG}^{+}$memory B cells isolated from asymptomatic $\mathrm{AID}^{+/-}$subjects, a feature correlating with decreased AID expression in their $\mathrm{B}$ cells upon activation (28). These data are also in agreement with the identification of increased microhomology sequences in switched regions of $\mathrm{AID}^{+/-}$memory $\mathrm{B}$ cells, a characteristic associated with less efficient CSR through decreased AID activity (56). Dominant AICDA mutations encoding hyperactive AID variants that cannot catalyze CSR also resulted in decreased SHM frequencies in $\operatorname{IgM}^{+}$ memory/marginal zone B cells (57). This paradoxical observation associating hyperactive AID enzyme activity with lower SHM may be reconciled with the possibility that hyperactive AID activity may induce exacerbated DNA lesions in GC B cells, causing B cells that attempt many rounds of antibody gene mutations to undergo apoptosis and be eliminated. Finally, 1 UNG-deficient patient who lacked CSR but had normal SHM frequency displayed GCs similar in size to those in the HD counterparts, further suggesting that SHM regulates $\mathrm{GC}$ responses.

In summary, our analysis of individuals with AICDA mutations revealed that decreased SHM processes lead to enhanced GC reactions and increased Tfh cell production and cytokine secretion, all of which likely result from the reduced efficiency and delayed kinetics of generating highly mutated antibodies that eliminate antigens from FDCs (Figure 8). These cytokines affect the polarization and composition of $\mathrm{T}$ cells in these patients and alter Treg function, which further results in a defective peripheral B cell tolerance checkpoint. This impaired selection step allows the accumulation of large numbers of autoreactive naive B cells, which are then activated by abundant Tfh cells and systemic cytokines, not only supporting ANA secretion but also the development of autoimmune manifestations. Hence, antibody replacement therapy in immunodeficient patients may be beneficial by providing mutated antibodies that dampen these abnormal immune features.

\section{Methods}

Patients and healthy donor controls. We obtained peripheral blood or frozen PBMCs from 21 AID-deficient patients with homozygous or compound heterozygous autosomal recessive AICDA mutations, 11 of whom were described previously (8). In addition, we collected samples from 16 healthy subjects related to these patients, who carried 1 mutated AICDA allele, as well as from $5 \mathrm{AD}$-AID patients and 5 UNG-deficient patients. Because of the lack of fresh PBMCs and the limited amount of frozen PBMCs or serum from these very rare patients, some experiments could not be performed on all samples. All patients' information is included in Supplemental Table 1. Agematched HDs were previously reported $(2,8,15,16,19,20,40)$.

Cell staining and sorting. Peripheral B cells from the blood of patients and control donors were purified by positive selection using CD2O-magentic beads (Miltenyi Biotec). Enriched B cells were stained with the following antibodies: anti-IgM-FITC (G20-127), anti-IgG-PE (G18-145), and anti-CD21-APC (clone B-ly4) from BD Biosciences; and anti-CD19-Pacific Blue (clone HIB19), anti-CD27-PercP-Cy5.5 (clone M-T271), and anti-CD10-PE-Cy7 (clone HI10a) from BioLegend. Single $\mathrm{CD}_{19}{ }^{+} \mathrm{CD} 21^{\mathrm{lo}} \mathrm{CD} 10^{+} \mathrm{IgM}^{\mathrm{hi}} \mathrm{CD} 27^{-}$new emigrant/transitional and $\mathrm{CD} 19^{+} \mathrm{CD} 21^{+} \mathrm{CD} 10^{-} \mathrm{IgM}^{+} \mathrm{CD} 27^{-}$mature naive $\mathrm{B}$ cells were sorted on a FACSAria Flow Cytometer (BD) into 96-well PCR plates and immediately frozen on dry ice. The following antibodies were used for T cell phenotyping: anti-CD4 APC-Cy7 (clone OKT4), anti-CD25 
PE-Cy7 (clone BC96), anti-CD127 PerCP-Cy5.5 (clone A019D5), anti-CXCR5 PerCP-Cy5.5 (clone J252D4), anti-PD-1 PE-Cy7 (clone EH12.2H7), and anti-ICOS APC (clone C398.4A) (all from BioLegend); anti-CD3 eFluor 605NC (clone OKT3; eBioscience); and antiHLA-DR APC (G46-6; BD Biosciences). Intracellular staining with anti-FOXP3 Alexa Fluor 488 (clone PCH101; eBioscience) and antiKi-67 PE (clone Ki-67; BioLegend) was performed using the FOXP3/ Transcription Factor Staining Buffer Set in accordance with the manufacturer's instructions (eBioscience).

cDNA, RT-PCR, antibody production and purification, ELISAs, and indirect immunofluorescence assays. Reverse transcription-PCR reactions, primer sequences, cloning strategy, expression vectors, recombinant antibody expression, purification, and reactivity determination were described previously (1). For indirect immunofluorescence assays (IFAs), HEp-2 cell-coated slides (Bion Enterprises Ltd.) were incubated in a moist chamber at room temperature with 1:80 and 1:320 diluted sera. FITC-conjugated monoclonal mouse anti-human IgM (Pharmingen, BD) was used as a detection reagent. For detection of cytokine transcripts, RNA was extracted from sorted CD4 ${ }^{+} \mathrm{T}$ cells using the Absolutely RNA Microprep Kit (Agilent Technologies), and 150 ng RNA was reverse transcribed using random hexamers (Applied Biosystems) and a SuperScript III Reverse Transcriptase Kit (Invitrogen, Thermo Fisher Scientific). The resulting cDNA was amplified in duplicate using Brilliant SYBR Green QPCR Master Mix (Agilent Technologies) and the Stratagene MX3005 real time detection system and normalized to CD4 for each sample before comparisons. Primer sets are summarized in Supplemental Table 2. Values are represented as the difference in Ct values normalized to HPRT1 for each sample.

Repertoire analysis. Ig sequences and mutation status were analyzed by Ig BLAST comparison with GenBank using the NCBI's IgBLAST server (http://www.ncbi.nlm.nih.gov/igblast/). Heavy chain complementarity determining region 3 was defined as the interval between amino acids at position 94 in the $V_{H}$ framework 3 and the conserved tryptophan at position 103 in JH segments. Sequences are shown in Supplemental Tables 3-16.

KREC assay. The ratio of KREC joints (signal joint) to the $\mathrm{J} \kappa-\mathrm{C} \kappa$ recombination genomic joints (coding joint) was determined as previously described (58). In brief, genomic DNA was isolated from sorted B cell fractions by lysing cell pellets in $10 \mathrm{mM}$ Tris- $\mathrm{HCl}, \mathrm{pH}$ 8.0, containing $100 \mu \mathrm{g} / \mathrm{ml}$ proteinase $\mathrm{K}$ (Roche), incubating for 1 hour at $56^{\circ} \mathrm{C}$, and heat inactivating the enzyme at $95^{\circ} \mathrm{C}$ for 10 minutes. Two separate RT-PCR reactions were performed, one reaction to amplify the signal joint and another to amplify the coding joint, as previously detailed (58). The number of cell divisions was calculated by subtracting the $\mathrm{Ct}$ of the PCR detecting the coding joint from that detecting the signal joint.

In vitro Treg suppression assay. $\mathrm{CD}^{+} \mathrm{T}$ cells were enriched using the EasySep Human CD4+ T Cell Enrichment Kit (STEMCELL Technologies). CD $4^{+} \mathrm{CD} 25^{\mathrm{hi}} \mathrm{CD} 127^{\mathrm{lo} /-}$ Tregs were sorted by flow cytometry, whereas $\mathrm{CD}^{+} \mathrm{CD}^{+} \mathrm{CD} 25^{-}$Tresps were obtained after the depletion of $\mathrm{CD} 25^{+}$cells with anti-human CD25 microbeads (Miltenyi Biotec) and then labeled with CellTrace CFSE (InvivoGen) at $5 \mu \mathrm{M}$. Tregs and Tresps were cocultured at a 1:1 ratio in the presence of beads loaded with anti-CD2, anti-CD3, and anti-CD28 (Treg Suppression Inspector, human; Miltenyi Biotec) at a 1 bead/1 cell ratio, with or without the addition of cytokines at $50 \mathrm{ng} / \mathrm{ml}$ concentration (Peprotech). On days 3.5 to 4.5 , cocultures were stained for viability with the LIVE/DEAD Kit (Invitrogen, Thermo Fisher Scientific), and proliferation of viable
Tresps was analyzed by CFSE dilution. For cytokine preincubation experiments, Treg populations were stimulated with $1 \mu \mathrm{g} / \mathrm{ml}$ platebound anti-CD3, $1 \mu \mathrm{g} / \mathrm{ml}$ soluble anti-CD28, and $25 \mathrm{U} / \mathrm{ml} \mathrm{IL-2} \mathrm{in} \mathrm{the}$ presence or absence of $50 \mathrm{ng} / \mathrm{ml} \mathrm{IL-4,} \mathrm{IL-10,} \mathrm{IL-17,} \mathrm{IL-21,} \mathrm{and} \mathrm{IFN-} \gamma$, $10 \mathrm{ng} / \mathrm{ml} \mathrm{IL-12,} \mathrm{or} 100 \mathrm{U} / \mathrm{ml} \mathrm{IL}-2$ for 4 days, washed twice, counted, and added to freshly isolated CFSE-labeled Tresps.

Cytokine detection. Plasma or serum BAFF concentrations were determined by ELISA according to the manufacturer's instructions (R\&D Systems). Cytokine plasma concentrations were measured with the High-Sensitivity Human Cytokine Magnetic Bead Kit (EMD Millipore) using a Luminex 200. IL-21 was measured by ELISA with the Human IL-21 ELISA Ready-Set-Go Kit (eBioscience). IL-17A was measured with the Duoset ELISA Kit (R\&D Systems). For intracellular cytokine detection, PBMCs were stimulated with $30 \mathrm{nM}$ PMA and $200 \mathrm{nM}$ ionomycin for 4 hours in the presence of GolgiStop (BD Biosciences), and intracellular staining of cytokines was performed using FOXP3 staining buffers (eBioscience) and the following antibodies: anti-IFN- $\gamma$ PE (clone 4S.B3; eBioscience); anti-IL-10 PE (clone JES319F1; BD Biosciences); anti-IL-4 PE (clone MP4-25D2), anti-IL-2 Alexa Fluor 647 (clone MQ17H12), anti-IL-17A Alexa Fluor 647 (clone BL168), and anti-IL-21 APC (clone 3A3N2) from BioLegend.

Statistics. Statistical analysis was performed using GraphPad Prism (version 5.0; GraphPad). Data are reported as the mean \pm SD. Differences between groups of research subjects were analyzed for statistical significance using either ANOVA with Dunnett's correction for testing multiple comparisons or unpaired, 2-tailed Student's $t$ tests. A $P$ value of 0.05 or less was considered significant.

Study approval. The human studies were reviewed and approved by the IRB of Yale University. All subjects provided informed consent prior to their participation in the study.

\section{Author contributions}

TC, JNS, TK, LM, and EM designed the experiments and discussed the data with AL, JEW, LDN, WAH, SSK, HDO, CCR, MVDB, TWK, SK, HK, YS, SN, and AD, who provided patients' samples and valuable research suggestions. TC, JNS, JMB, YSN, CM, FRD, NY, SG, NC, TK, LM, and JB performed the experiments, with help from EM for cell isolation and sorting. TC and EM wrote the manuscript.

\section{Acknowledgments}

We are very much indebted to the patients and their families. We thank all the members of the Immunodeficiency Clinic of the Centre Hospitalier de l'Université Laval, as well as S. Rudchenko, L. Devine and C. Wang for cell sorting. This work was supported by grants from the National Institute of Allergy and Infectious Diseases (NIH/NIAID), NIH (AI071087, AI082713, and AI095848, to E. Meffre; AI061093, to EM and CCR; and T32 AI089704, to LM). TC received support from the Rubicon Program of the Netherlands Organization for Scientific Research. TK was supported by the Sigrid Juselius Foundation, the Finnish Medical Foundation, and the Saastamoinen Foundation. HDO is supported by the Jeffrey Modell Foundation. SK is a researcher at the Centre National de la Recherche Scientifique (CNRS) (Paris, France).

Address correspondence to: Eric Meffre, Yale University School of Medicine, 300 George Street, Room 353F, New Haven, Connecticut 06511, USA. Phone: 203.737.4535; E-mail: Eric.meffre@yale.edu. 
1. Wardemann H, Yurasov S, Schaefer A, Young JW, Meffre E, Nussenzweig MC. Predominant autoantibody production by early human B cell precursors. Science. 2003;301(5638):1374-1377.

2. Kinnunen T, et al. Accumulation of peripheral autoreactive $B$ cells in the absence of functional human regulatory T cells. Blood. 2013;121(9):1595-1603.

3. Imai K, et al. Human uracil-DNA glycosylase deficiency associated with profoundly impaired immunoglobulin class-switch recombination. Nat Immunol. 2003;4(10):1023-1028.

4. Lee WI, Torgerson TR, Schumacher MJ, Yel L, Zhu Q, Ochs HD. Molecular analysis of a large cohort of patients with the hyper immunoglobulin M (IgM) syndrome. Blood. 2005;105(5):1881-1890.

5. Muramatsu M, Kinoshita K, Fagarasan S, Yamada S, Shinkai Y, Honjo T. Class switch recombination and hypermutation require activation-induced cytidine deaminase (AID), a potential RNA editing enzyme. Cell. 2000;102(5):553-563.

6. Revy P, et al. Activation-induced cytidine deaminase (AID) deficiency causes the autosomal recessive form of the Hyper-IgM syndrome (HIGM2). Cell. 2000;102(5):565-575.

7. Hervé M, et al. CD4O ligand and MHC class II expression are essential for human peripheral $\mathrm{B}$ cell tolerance. JExp Med. 2007;204(7):1583-1593.

8. Meyers G, et al. Activation-induced cytidine deaminase (AID) is required for $\mathrm{B}$-cell tolerance in humans. Proc Natl Acad Sci U S A. 2011;108(28):11554-11559.

9. Aruffo A, et al. The CD40 ligand, gp39, is defective in activated $\mathrm{T}$ cells from patients with X-linked hyper-IgM syndrome. Cell. 1993;72(2):291-300.

10. Imai K, et al. Analysis of class switch recombination and somatic hypermutation in patients affected with autosomal dominant hyper-IgM syndrome type 2. Clin Immunol. 2005;115(3):277-285.

11. Ito $S$, et al. Activation-induced cytidine deaminase shuttles between nucleus and cytoplasm like apolipoprotein $B$ mRNA editing catalytic polypeptide 1. Proc Natl Acad Sci US A. 2004;101(7):1975-1980.

12. Silberstein LE, et al. Variable region gene analysis of pathologic human autoantibodies to the related $\mathrm{i}$ and I red blood cell antigens. Blood. 1991;78(9):2372-2386.

13. Pascual V, Cha S, Gershwin ME, Capra JD, Leung PS. Nucleotide sequence analysis of natural and combinatorial anti-PDC-E2 antibodies in patients with primary biliary cirrhosis. Recapitulating immune selection with molecular biology. JImmunol. 1994;152(5):2577-2585.

14. Meffre E. The establishment of early B cell tolerance in humans: lessons from primary immunodeficiency diseases. Ann N Y Acad Sci. 2011;1246:1-10.

15. Menard L, et al. Signaling lymphocytic activation molecule (SLAM)/SLAM-associated protein pathway regulates human B-cell tolerance. JAllergy Clin Immunol. 2014;133(4):1149-1161.

16. Romberg N, et al. CVID-associated TACI mutations affect autoreactive $\mathrm{B}$ cell selection and activation. JClin Invest. 2013;123(10):4283-4293.
17. Mackay F, Schneider P. Cracking the BAFF code. Nat Rev Immunol. 2009;9(7):491-502.

18. Janssen E, et al. Dedicator of cytokinesis 8-deficient patients have a breakdown in peripheral $\mathrm{B}$-cell tolerance and defective regulatory $\mathrm{T}$ cells. J Allergy Clin Immunol. 2014;134(6):1365-1374.

19. Isnardi I, et al. Complement receptor $2 /$ CD21- human naive B cells contain mostly autoreactive unresponsive clones. Blood. 2010;115(24):5026-5036.

20. Pala F, et al. Lentiviral-mediated gene therapy restores B cell tolerance in WiskottAldrich syndrome patients. J Clin Invest. 2015;125(10):3941-3951.

21. Peluso I, et al. IL-21 counteracts the regulatory T cell-mediated suppression of human CD4+ T lymphocytes. J Immunol. 2007;178(2):732-739.

22. Dominguez-Villar M, Baecher-Allan CM, Hafler DA. Identification of T helper type 1-like, Foxp3+ regulatory $\mathrm{T}$ cells in human autoimmune disease. Nat Med.2011;17(6):673-675.

23. Maloy KJ, Powrie F. Fueling regulation: IL-2 keeps CD4+ Treg cells fit. Nat Immunol. 2005;6(11):1071-1072.

24. Locci M, et al. Human circulating PD-1+ CXCR3-CXCR5+ memory Tfh cells are highly functional and correlate with broadly neutralizing HIV antibody responses. Immunity. 2013;39(4):758-769.

25. Morita R, et al. Human blood CXCR5(+) $\mathrm{CD} 4(+) \mathrm{T}$ cells are counterparts of $\mathrm{T}$ follicular cells and contain specific subsets that differentially support antibody secretion. Immunity. 2011;34(1):108-121.

26. McBride KM, Gazumyan A, Woo EM, Schwickert TA, Chait BT, Nussenzweig MC. Regulation of class switch recombination and somatic mutation by AID phosphorylation. J Exp Med. 2008;205(11):2585-2594.

27. Takizawa M, et al. AID expression levels determine the extent of $\mathrm{cMyc}$ oncogenic translocations and the incidence of $\mathrm{B}$ cell tumor development. J Exp Med. 2008;205(9):1949-1957.

28. Cantaert T, et al. Activation-induced cytidine deaminase expression in human $B$ cell precursors is essential for central B cell tolerance. Immunity. 2015;43(5):884-895.

29. Tsuiji M, Yurasov S, Velinzon K, Thomas S, Nussenzweig MC, Wardemann H. A checkpoint for autoreactivity in human IgM+ memory B cell development. J Exp Med. 2006;203(2):393-400.

30. Tiller T, Tsuiji M, Yurasov S, Velinzon K, Nussenzweig MC, Wardemann H. Autoreactivity in human IgG+ memory B cells. Immunity. 2007;26(2):205-213.

31. Durandy A, Cantaert T, Kracker S, Meffre E. Potential roles of activation-induced cytidine deaminase in promotion or prevention of autoimmunity in humans. Autoimmunity. 2013;46(2):148-156.

32. Kuraoka M, et al. Activation-induced cytidine deaminase mediates central tolerance in B cells. Proc Natl Acad Sci U S A. 2011;108(28):11560-11565.

33. Lesley R, et al. Reduced competitiveness of autoantigen-engaged $\mathrm{B}$ cells due to increased dependence on BAFF. Immunity. 2004;20(4):441-453.

34. Thien M, et al. Excess BAFF rescues self-reactive
$\mathrm{B}$ cells from peripheral deletion and allows them to enter forbidden follicular and marginal zone niches. Immunity. 2004;20(6):785-798.

35. Navarra SV, et al. Efficacy and safety of belimumab in patients with active systemic lupus erythematosus: a randomised, placebo-controlled, phase 3 trial. Lancet. 2011;377(9767):721-731.

36. Yurasov $S$, et al. Persistent expression of autoantibodies in SLE patients in remission. J Exp Med. 2006;203(10):2255-2261.

37. Yurasov S, et al. Defective B cell tolerance checkpoints in systemic lupus erythematosus. JExp Med. 2005;201(5):703-711.

38. Cunningham-Rundles C, Bodian C. Common variable immunodeficiency: clinical and immunological features of 248 patients. Clin Immunol. 1999;92(1):34-48.

39. Gulino AV, Notarangelo LD. Hyper IgM syndromes. Curr Opin Rheumatol. 2003;15(4):422-429.

40. Kinnunen T, et al. Specific peripheral B cell tolerance defects in patients with multiple sclerosis. JClin Invest. 2013;123(6):2737-2741.

41. Jacobi AM, et al. Activated memory B cell subsets correlate with disease activity in systemic lupus erythematosus: delineation by expression of CD27, IgD, and CD95. Arthritis Rheum. 2008;58(6):1762-1773.

42. Mackay F, Silveira PA, Brink R. B cells and the BAFF/APRIL axis: fast-forward on autoimmunity and signaling. Curr Opin Immunol. 2007;19(3):327-336.

43. Sellam J, et al. Blood memory B cells are disturbed and predict the response to rituximab in patients with rheumatoid arthritis. Arthritis Rheum. 2011;63(12):3692-3701.

44. Anderson SM, Tomayko MM, Ahuja A, Haberman AM, Shlomchik MJ. New markers for murine memory B cells that define mutated and unmutated subsets. J Exp Med. 2007;204(9):2103-2114.

45. Avery DT, et al. B cell-intrinsic signaling through IL-21 receptor and STAT3 is required for establishing long-lived antibody responses in humans. JExp Med. 2010;207(1):155-171.

46. Recher M, et al. IL-21 is the primary common $\gamma$ chain-binding cytokine required for human B-cell differentiation in vivo. Blood. 2011;118(26):6824-6835.

47. Johnston RJ, et al. Bcl6 and Blimp-1 are reciprocal and antagonistic regulators of $\mathrm{T}$ follicular helper cell differentiation. Science. 2009;325(5943):1006-1010.

48. Honjo T, Kinoshita K, Muramatsu M. Molecular mechanism of class switch recombination: linkage with somatic hypermutation. Annu Rev Immunol. 2002;20:165-196.

49. Liu YJ, Joshua DE, Williams GT, Smith CA, Gordon J, MacLennan IC. Mechanism of antigen-driven selection in germinal centres. Nature. 1989;342(6252):929-931.

50. Shlomchik MJ, Weisel F. Germinal center selection and the development of memory B and plasma cells. Immunol Rev. 2012;247(1):52-63.

51. Zhang Y, et al. Germinal center B cells govern their own fate via antibody feedback. J Exp Med. 2013;210(3):457-464.

52. Pape KA, Taylor JJ, Maul RW, Gearhart PJ, Jenkins MK. Different B cell populations 


\section{RESEARCH ARTICLE}

mediate early and late memory during an endogenous immune response. Science. 2011;331(6021):1203-1207.

53. Zaheen A, Boulianne B, Parsa JY, Ramachandran $\mathrm{S}$, Gommerman JL, Martin A. AID constrains germinal center size by rendering $B$ cells susceptible to apoptosis. Blood. 2009;114(3):547-554.

54. Wei M, Shinkura R, Doi Y, Maruya M, Fagarasan S, Honjo T. Mice carrying a knock-in mutation of Aicda resulting in a defect in somatic hypermutation have impaired gut homeostasis and compromised mucosal defense. Nat Immunol. 2011;12(3):264-270.

55. Fagarasan S, Muramatsu M, Suzuki K, Nagaoka $\mathrm{H}$, Hiai H, Honjo T. Critical roles of activationinduced cytidine deaminase in the homeostasis of gut flora. Science. 2002;298(5597):1424-1427.

56. Kracker S, Imai K, Gardès P, Ochs HD, Fischer A, Durandy AH. Impaired induction of DNA lesions during immunoglobulin classswitch recombination in humans influences end-joining repair. Proc Natl Acad Sci US A.
The Journal of Clinical Investigation

2010;107(51):22225-22230.

57. Zahn A, et al. Activation induced deaminase C-terminal domain links DNA breaks to end protection and repair during class switch recombination. Proc Natl Acad Sci US A. 2014;111(11):E988-E997.

58. van Zelm MC, Szczepanski T, van der Burg M, van Dongen JJ. Replication history of B lymphocytes reveals homeostatic proliferation and extensive antigen-induced B cell expansion. JExp Med. 2007;204(3):645-655. 